J. Elias, L. Robbiano and G. Valla

Nagoya Math. J.

Vol. 123 (1991), 39-76

\title{
NUMBER OF GENERATORS OF IDEALS
}

\author{
JUAN ELIAS ${ }^{1)}$, LORENZO ROBBIANO ${ }^{2)}$ \\ AND GIUSEPPE VALLA
}

\section{Dedicated to Professor Hideyuki Matsumura on his sixtieth birthday}

\section{Introduction}

Let $I$ be a homogeneous ideal of a polynomial ring over a field, $\nu(I)$ the number of elements of any minimal basis of $I, e=e(I)$ the multiplicity or degree of $R / I, h=h(I)$ the height or codimension of $I, i=\operatorname{indeg}(I)$ the initial degree of $I$, i.e. the minimal degree of non zero elements of $I$.

This paper is mainly devoted to find bounds for $\nu(I)$ when $I$ ranges over large classes of ideals. For instance we get bounds when $I$ ranges over the set of perfect ideals with preassigned codimension and multiplicity and when $I$ ranges over the set of perfect ideals with preassigned codimension, multiplicity and initial degree. Moreover all the bounds are sharp since they are attained by suitable ideals. Now let us make some historical remarks.

It is a classical result of Krull that $h(I) \leq \nu(I)$, and Macaulay showed that there is no upper bound for $\nu(I)$ when $I$ ranges over the set of perfect codimension 2 prime ideals of $k[x, y, z]$. But what happens if $e(I)$ is given? Many authors studied a more general problem, allowing the ambient ring $R$ to be a Cohen-Macaulay ring. If $R$ is specialized to be a polynomial ring or a regular local ring, we deduce from their results the following bounds:

$$
\begin{array}{ll}
\nu \leq e^{h-1}+h-1 & \text { Sally (1976) } \\
\nu \leq(h ! / \sqrt[h]{h !}) e^{1-1 / h}+h-1 & \text { Boratynski-Eisenbud-Rees (1979) } \\
\nu \leq 1+\left[(h-1)^{2} / h\right] e+\left(h^{2}-1\right) / h-\left(\begin{array}{c}
h \\
2
\end{array}\right) \quad \text { Valla (1981) }
\end{array}
$$

Received April 4, 1990.

1) The first author was partly supported by the Department of Mathematics of the University of Genova.

2) The second author was partly supported by the U.S. Army Research Office through the Mathematical Sciences Institute of Cornell University. 
Other results of Briançon-Iarrobino (1978) and Berman (1981) deal with the asymptotic behaviour of $\nu(I)$. For some historical background about this kind of problems, a good reference is the book of Sally (1978).

Now we come to a more detailed description of our paper. In the first section we collect many properties about binomial representations and some operations on them. All these results are elementary and more or less well-known; we included them for the sake of completeness, since they are used throughout the paper as technical tools.

Then we recall some fundamental theorems, which allow us to study special ideals called lex-segment ideals. To every given homogeneous ideal $I$ in the polynomial ring one can associate a suitable lex-segment ideal $J$ with the same Hilbert function, and the first remark is that $\nu(I)$ $\leq \nu(J)$ (see Corollary 2.7); the main observation of the second section is that $\nu(J)$ can be computed as the sum of the multiplicities of a suitable chain of hyperplane sections of $J$ (see Theorem 2.9 and Corollary 2.10).

Then after many preparatory Lemmas, we prove Main Lemma 3.9, from which we deduce Theorem 3.10 and Corollary 3.11. These results are the technical core of the paper in the following sense: given a family $\mathscr{F}$ of ideals, they yield a strategy for selecting a suitable lex-segment ideal $J(\mathscr{F})$ with the maximum number of generators among the members of $\mathscr{F}$. When $\mathscr{F}$ is the family of homogeneous perfect ideals with given $e, h$, then the ideal $J(\mathscr{F})$ turns out to be the same as in Berman's paper, but here we face a more subtle situation when we work with the family of homogeneous perfect ideals with given $e, h$ and indeg (see for instance Example 1 of Section 4). We can detect all these "extremal" ideals $J$ and we can compute $\nu(J)$ in all cases (see Proposition 4.2, Proposition 4.4 and Proposition 4.6) and Section 4 ends with the two main theorems, namely Theorem 4.7 and Theorem 4.8; they give the answer to the problem of finding sharp bounds for $\nu(I)$ inside many important families of zerodimensional ideals in the polynomial ring; moreover such bounds are explicitly given and many relations among them are described.

Section 5 is devoted to the study of several applications of our results. The first one deals with the extension of the preceding results to perfect ideals in regular local rings; it turns out that the same bounds hold. For codimension 2 ideals we give a simple proof of the well-known inequality $\nu(I) \leq$ indeg $(I)+1$, which avoids the use of Hilbert-Burch theorem. Then we prove that if a zero-dimensional ideal $I$ in a regular local ring 
$(R, \mathfrak{m})$ has the same multiplicity and the same number of generators as $\mathrm{m}^{t}$, then $I=\mathfrak{m}^{t}$ (Proposition 5.3).

Another application is shown to the asymptotic behaviour of $\nu(J)$ inside some classes of perfect ideals, when we let $e(I)$ tend to infinity. Here we generalize Berman's results in many directions (see Theorem 5.4).

In Proposition 5.5 we prove that all the preceding bounds can be attained by radical ideals, namely by the defining ideals of the scheme associated to sets of points in the projective space.

We conclude by sketching some applications of our results to the size of Gröbner Bases of zero-dimensional ideals in the polynomial rings.

Finally we enclose two tables at the end of the paper, where some of the bounds are displayed. All the computations were performed by CoCoA, a Computational Commutative Algebra System under development at the University of Genova.

\section{Index}

Introduction

1. Binomial representations and their arithmetic

2. Theorems of Macaulay, Stanley, Green and Lex-segments

3. Main Tools

4. Main Theorems

5. Applications

6. Tables

\section{§1. Binomial representations and their arithmetic}

We make the following conventions:

$$
\left(\begin{array}{c}
m \\
0
\end{array}\right)=1 \text { if } m \geq 1, \text { and }\left(\begin{array}{c}
m \\
k
\end{array}\right)=0 \text { if } m<k
$$

We recall that if $n$ and $i$ are positive integers then $n$ can be uniquely written as

$$
n=\left(\begin{array}{c}
n(i) \\
i
\end{array}\right)+\left(\begin{array}{c}
n(i-1) \\
i-1
\end{array}\right)+\cdots+\left(\begin{array}{c}
n(j) \\
j
\end{array}\right)
$$

where $n(i)>n(i-1)>\cdots>n(j) \geq j \geq 1$.

This is called the $i$-binomial expansion of $n$.

Property 1.1. Let $m=\sum_{k=p}^{i}\left(\begin{array}{c}m(k) \\ k\end{array}\right)$ and $n=\sum_{k=j}^{i}\left(\begin{array}{c}n(k) \\ k\end{array}\right)$ be positive 
integers; then $m>n$ if and only if $\{m(i), m(i-1), \cdots, m(p)\}>\{n(i), n(i-1)$, $\cdots, n(j)\}$ in the lexicographic ordering.

Proof. The assertion is clear from the definition of the $i$-binomial expansion of any positive integer

We let

$$
\begin{array}{ll}
\text { *) } & n^{\langle i\rangle}:=\left(\begin{array}{c}
n(i)+1 \\
i+1
\end{array}\right)+\left(\begin{array}{c}
n(i-1)+1 \\
i
\end{array}\right)+\cdots+\left(\begin{array}{c}
n(j)+1 \\
j+1
\end{array}\right) \\
(* *) & n_{\langle i\rangle}:=\left(\begin{array}{c}
n(i)-1 \\
i
\end{array}\right)+\left(\begin{array}{c}
n(i-1)-1 \\
i-1
\end{array}\right)+\cdots+\left(\begin{array}{c}
n(j)-1 \\
j
\end{array}\right)
\end{array}
$$

and we remark that $\left(^{*}\right)$ is the $(i+1)$-binomial expansion of $n^{\langle i\rangle}$ while $(* *)$ is the $i$-binomial expansion of $n_{\langle i\rangle}$ iff $n(j)>j$.

Property 1.2.

$$
(n+1)^{\langle i\rangle}=n^{\langle i\rangle}+1+n(1)
$$

where if $j>1$ we let $n(1)=0$.

Proof. If $j>1$ we have

$$
n+1=\left(\begin{array}{c}
n(i) \\
i
\end{array}\right)+\left(\begin{array}{c}
n(i-1) \\
i-1
\end{array}\right)+\cdots+\left(\begin{array}{c}
n(j) \\
j
\end{array}\right)+\left(\begin{array}{l}
j-1 \\
j-1
\end{array}\right)
$$

hence

$$
\begin{aligned}
(n+1)^{\langle i\rangle} & =\left(\begin{array}{c}
n(i)+1 \\
i+1
\end{array}\right)+\left(\begin{array}{c}
n(i-1)+1 \\
i
\end{array}\right)+\cdots+\left(\begin{array}{c}
n(j)+1 \\
j+1
\end{array}\right)+\left(\begin{array}{l}
j \\
j
\end{array}\right) \\
& =n^{\langle i\rangle}+1 .
\end{aligned}
$$

If $j=1$, let $s$ be the maximum integer such that $n(s)=n(1)+s-1$. Then $1 \leq s \leq i$ and we get

$$
\begin{aligned}
n & =\left(\begin{array}{c}
n(i) \\
i
\end{array}\right)+\left(\begin{array}{c}
n(i-1) \\
i-1
\end{array}\right)+\cdots+\left(\begin{array}{c}
n(s+1) \\
s+1
\end{array}\right)+\sum_{k=1}^{s}\left(\begin{array}{c}
n(1)+k-1 \\
k
\end{array}\right) \\
& =\left(\begin{array}{c}
n(i) \\
i
\end{array}\right)+\left(\begin{array}{c}
n(i-1) \\
i-1
\end{array}\right)+\cdots+\left(\begin{array}{c}
n(s+1) \\
s+1
\end{array}\right)+\left(\begin{array}{c}
n(1)+s \\
s
\end{array}\right)-1
\end{aligned}
$$

hence

$$
n+1=\left(\begin{array}{c}
n(i) \\
i
\end{array}\right)+\left(\begin{array}{c}
n(i-1) \\
i-1
\end{array}\right)+\cdots+\left(\begin{array}{c}
n(s+1) \\
s+1
\end{array}\right)+\left(\begin{array}{c}
n(1)+s \\
s
\end{array}\right)
$$

is the $i$-binomial expansion of $n+1$, since $n(1)+s<n(s+1)$. We get 


$$
\begin{aligned}
n^{\langle i\rangle} & =\left(\begin{array}{c}
n(i)+1 \\
i+1
\end{array}\right)+\cdots+\left(\begin{array}{c}
n(s+1)+1 \\
s+2
\end{array}\right)+\sum_{k=1}^{s}\left(\begin{array}{c}
n(1)+k \\
k+1
\end{array}\right) \\
& =\left(\begin{array}{c}
n(i)+1 \\
i+1
\end{array}\right)+\cdots+\left(\begin{array}{c}
n(s+1)+1 \\
s+2
\end{array}\right)+\sum_{r=2}^{s+1}\left(\begin{array}{c}
n(1)+r-1 \\
r
\end{array}\right) \\
& =\left(\begin{array}{c}
n(i)+1 \\
i+1
\end{array}\right)+\cdots+\left(\begin{array}{c}
n(s+1)+1 \\
s+2
\end{array}\right)+\left(\begin{array}{c}
s+1+n(1) \\
n(1)
\end{array}\right)-1-n(1)
\end{aligned}
$$

and

$$
(n+1)^{\langle i\rangle}=\left(\begin{array}{c}
n(i)+1 \\
i+1
\end{array}\right)+\cdots+\left(\begin{array}{c}
n(s+1)+1 \\
s+2
\end{array}\right)+\left(\begin{array}{c}
s+1+n(1) \\
s+1
\end{array}\right)
$$

the conclusion follows

Property 1.3.

$$
(n+1)_{\langle i\rangle}= \begin{cases}n_{\langle i\rangle}, & \text { if } j>1 \\ n_{\langle i\rangle}+1, & \text { if } j=1 .\end{cases}
$$

Proof. If $j>1$ we get

$$
n+1=\left(\begin{array}{c}
n(i) \\
i
\end{array}\right)+\cdots+\left(\begin{array}{c}
n(j) \\
j
\end{array}\right)+\left(\begin{array}{c}
j-1 \\
j-1
\end{array}\right)
$$

hence

$$
(n+1)_{\langle i\rangle}=\left(\begin{array}{c}
n(i)-1 \\
i
\end{array}\right)+\cdots+\left(\begin{array}{c}
n(j)-1 \\
j
\end{array}\right)=n_{\langle i\rangle}
$$

If $j=1$, let $s$ be the maximum integer such that $n(s)=n(1)+s-1$. Then $1 \leq s \leq i$ and we get as before

$$
n+1=\left(\begin{array}{c}
n(i) \\
i
\end{array}\right)+\cdots+\left(\begin{array}{c}
n(s+1) \\
s+1
\end{array}\right)+\left(\begin{array}{c}
n(1)+s \\
s
\end{array}\right)
$$

hence

$$
(n+1)_{\langle i\rangle}=\left(\begin{array}{c}
n(i)-1 \\
i
\end{array}\right)+\cdots+\left(\begin{array}{c}
n(s+1)-1 \\
s+1
\end{array}\right)+\left(\begin{array}{c}
n(1)+s-1 \\
s
\end{array}\right)
$$

and

$$
\begin{aligned}
n_{\langle i\rangle} & =\left(\begin{array}{c}
n(i)-1 \\
i
\end{array}\right)+\cdots+\left(\begin{array}{c}
n(s+1)-1 \\
s+1
\end{array}\right)+\sum_{k=1}^{s}\left(\begin{array}{c}
n(1)+k-2 \\
k
\end{array}\right) \\
& =\left(\begin{array}{c}
n(i)-1 \\
i
\end{array}\right)+\cdots+\left(\begin{array}{c}
n(s+1)-1 \\
s+1
\end{array}\right)+\left(\begin{array}{c}
s+n(1)-1 \\
s
\end{array}\right)-1 .
\end{aligned}
$$

This proves our assertion. 
Given positive numbers $n$ and $i$ we let

$$
n(i)_{-1}:=n-n_{\langle i\rangle}
$$

and we write $n_{-1}$ if there is no ambiguity.

Property 1.4.

$$
n_{-1}=\left(\begin{array}{c}
n(i)-1 \\
i-1
\end{array}\right)+\cdots+\left(\begin{array}{c}
n(j)-1 \\
j-1
\end{array}\right)
$$

Proof. We have

$$
n-n_{\langle i\rangle}=\sum_{k=j}^{i}\left(\begin{array}{c}
n(k) \\
k
\end{array}\right)-\sum_{k=j}^{i}\left(\begin{array}{c}
n(k)-1 \\
k
\end{array}\right)=\sum_{k=j}^{i}\left(\begin{array}{c}
n(k)-1 \\
k-1
\end{array}\right)
$$

which concludes the proof

We need also to define $0_{\langle i\rangle}=0^{\langle i\rangle}=0, \forall i \geq 1$.

PROPERTy 1.5. If $n \geq m>0$ we have $n-n_{\langle i\rangle}=n_{-1} \geq m-m_{\langle i\rangle}=m_{-1}$.

Proof. By Property 1.3 it is clear that $n_{\langle i\rangle} \leq m_{\langle i\rangle}+n-m$, hence the conclusion follows.

Property 1.6. For all non negative integers $n$ we have

$$
\left(n^{\langle i\rangle}\right)_{\langle i+1\rangle}=\left(n_{\langle i\rangle}\right\rangle^{\langle i\rangle}=n^{\langle i\rangle}-n .
$$

Proof. Namely

$$
n^{\langle i\rangle}-n=\sum_{k=j}^{i}\left(\left(\begin{array}{c}
n(k)+1 \\
k+1
\end{array}\right)-\left(\begin{array}{c}
n(k) \\
k
\end{array}\right)\right)=\sum_{k=j}^{i}\left(\begin{array}{c}
n(k) \\
k+1
\end{array}\right)
$$

and also

$$
\left(n^{\langle i\rangle}\right)_{\langle i+1\rangle}=\left(\sum_{k=j}^{i}\left(\begin{array}{c}
n(k)+1 \\
k+1
\end{array}\right)\right)_{\langle i+1\rangle}=\sum_{k=j}^{i}\left(\begin{array}{c}
n(k) \\
k+1
\end{array}\right) .
$$

Now if $n(j)>j$ we get

$$
\left(n_{\langle i\rangle}{ }^{\langle i\rangle}=\left(\sum_{k=j}^{i}\left(\begin{array}{c}
n(k)-1 \\
k
\end{array}\right)\right)^{\langle i\rangle}=\sum_{k=j}^{i}\left(\begin{array}{c}
n(k) \\
k+1
\end{array}\right)=n^{\langle i\rangle}-n .\right.
$$

If $n(j)=j$, let $s$ be the maximum integer such that $n(s)=s$. Then $j \leq s \leq i$ and if we let $b=n-s$, we have $n^{\langle i\rangle}=b^{\langle i\rangle}+s, n_{\langle i\rangle}=b_{\langle i\rangle}$ and $b^{\langle i\rangle}-b=\left(b_{\langle i\rangle}\right)^{\langle i\rangle}$. It follows $n^{\langle i\rangle}-n=b^{\langle i\rangle}-b=\left(b_{\langle i\rangle}\right)^{\langle i\rangle}=\left(n_{\langle i\rangle}\right)^{\langle i\rangle}$

Property 1.7. Foe every $i \geq 2$ and every positive integer $n$ we have 


$$
\left(n-n_{\langle i\rangle}\right)^{\langle i-1\rangle}= \begin{cases}n & \text { if } j>1 \\ n+n(2)-n(1) & \text { if } j=1 .\end{cases}
$$

Proof. If $j>1$, then

$$
n-n_{\langle i\rangle}=\sum_{k=j}^{i}\left[\left(\begin{array}{c}
n(k) \\
k
\end{array}\right)-\left(\begin{array}{c}
n(k)-1 \\
k
\end{array}\right)\right]=\sum_{k=j}^{i}\left(\begin{array}{c}
n(k)-1 \\
k-1
\end{array}\right)
$$

is the $(i-1)$-th binomial expansion of $n-n_{\langle i\rangle}$, hence

$$
\left(n-n_{\langle i\rangle}\right)^{\langle i-1\rangle}=\sum_{k=j}^{i}\left(\begin{array}{c}
n(k) \\
k
\end{array}\right)=n
$$

If $j=1$, then $n-n_{\langle i\rangle}=c+1$, where $c:=\sum_{k=2}^{i}\left(\begin{array}{c}n(k)-1 \\ k-1\end{array}\right) . \quad$ Using Property 1.2 we get

$$
\left(n-n_{\langle i\rangle}\right)^{\langle i-1\rangle}=(c+1)^{\langle i-1\rangle}=c^{\langle i\rangle}+1+n(2)-1=n-n(1)+n(2)
$$

and the conclusion follows

Property 1.8. If $n \leq m^{\langle i-1\rangle}$, then $n-n_{\langle i\rangle}=n_{-1} \leq m$ for every $i \geq 2$.

Proof. If $j>1$, then $n=\left[n-n_{\langle i\rangle}\right]^{\langle i-1\rangle} \leq m^{\langle i-1\rangle}$ and the conclusion follows by Property 1.1. If $j=1$, then as before we have $n-n_{\langle i\rangle}=c+1$, where $c:=\sum_{k=2}^{i}\left(\begin{array}{c}n(k)-1 \\ k-1\end{array}\right)$. Thus if $m<c+1$ we get by Property 1.1 that $m^{\langle i-1\rangle} \leq c^{\langle i-1\rangle}=n-n(1)<n$, a contradiction.

In the following we say that $n$ is $i$-regular if $n(k)=n(j)+k-j$ for every $k=j, \cdots, i$.

PROPERTY 1.9. If $n$ is $i$-regular and $n>i$, then $n_{\langle i\rangle}$ is $i$-regular.

Proof. Since $n>1$ and $n$ is $i$-regular, $n(j)>j$, hence the $i$-binomial expansion of $n_{\langle i\rangle}$ is $n_{\langle i\rangle}=\sum_{k=j}^{i}\left(\begin{array}{c}n(k)-1 \\ k\end{array}\right)$. The conclusion follows

PROPERTy 1.10. If $n$ is $i$-regular, then $n-n_{\langle i\rangle}$ is $(i-1)$-regular.

Proof. Since $n-n_{\langle i\rangle}=\sum_{k=j}^{i}\left(\begin{array}{c}n(k)-1 \\ k-1\end{array}\right)$ the result it clear if $j>1$. If $j=1$, then we have $n-n_{\langle i\rangle}=\sum_{k=1}^{i}\left(\begin{array}{c}n(k)-1 \\ k-1\end{array}\right)=\sum_{k=2}^{i}\left(\begin{array}{c}n(1)+k-1-1 \\ k-1\end{array}\right)$ $+1=\sum_{s=0}^{i=1}\left(\begin{array}{c}n(1)+s-1 \\ s\end{array}\right)=\left(\begin{array}{c}n(1)+i-1 \\ i-1\end{array}\right)$

Property 1.11. Let $j=1$ and $p$ be the maximum integer such that 
$n(p)=n(2)+p-2$. Then $p \geq 2$ and we have the following $(i-1)$-expansion of $n_{-1}$.

$$
n_{-1}=n-n_{\langle i\rangle}=\left(\begin{array}{c}
n(i)-1 \\
i-1
\end{array}\right)+\cdots+\left(\begin{array}{c}
n(p+1)-1 \\
p
\end{array}\right)+\left(\begin{array}{c}
n(2)+p-2 \\
p-1
\end{array}\right)
$$

Proof. We have

$$
\begin{aligned}
n-n_{\langle i\rangle} & =\sum_{k=2}^{i}\left(\begin{array}{c}
n(k)-1 \\
k-1
\end{array}\right)+1 \\
& =\sum_{k=p+1}^{i}\left(\begin{array}{c}
n(k)-1 \\
k-1
\end{array}\right)+\sum_{k=2}^{p}\left(\begin{array}{c}
n(2)+k-3 \\
k-1
\end{array}\right)+1 \\
& =\sum_{k=p+1}^{i}\left(\begin{array}{c}
n(k)-1 \\
k-1
\end{array}\right)+\sum_{m=0}^{p-1}\left(\begin{array}{c}
n(2)+m-2 \\
m
\end{array}\right) \\
& =\sum_{k=p+1}^{i}\left(\begin{array}{c}
n(k)-1 \\
k-1
\end{array}\right)+\left(\begin{array}{c}
n(2)+p-2 \\
p-1
\end{array}\right) .
\end{aligned}
$$

This proves our assertion.

We define $\delta_{i}(n):=n(i)-i$ and remark that $\delta_{i}(n) \geq n(k)-k$ for every $k=j, \cdots, i$.

We define $n_{\langle i\rangle(0)}:=n$ and inductively $n_{\langle i\rangle(t)}:=\left(n_{\langle i\rangle(t-1)}\right)_{\langle i\rangle}$.

Property 1.12. For all $r \geq \delta_{i}(n)$ we have

$$
n^{\langle i\rangle}=\sum_{t=0}^{r} n_{\langle i\rangle(t)}
$$

Proof. We have $n^{\langle i\rangle}=\sum_{k=j}^{i}\left(\begin{array}{c}n(k)+1 \\ k+1\end{array}\right)$ and $n_{\langle i\rangle(t)}=\sum_{k=j}^{i}\left(\begin{array}{c}n(k)-t \\ k\end{array}\right)$.

Hence we get

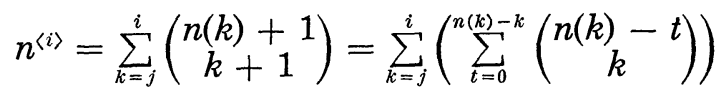

$$
\begin{aligned}
& =\sum_{k=j}^{i}\left(\sum_{t=0}^{r}\left(\begin{array}{c}
n(k)-t \\
k
\end{array}\right)\right)=\sum_{t=0}^{r}\left(\sum_{k=j}^{i}\left(\begin{array}{c}
n(k)-t \\
k
\end{array}\right)\right)=\sum_{t=0}^{r} n_{\langle i\rangle(t)}
\end{aligned}
$$

as wanted

Property 1.13. Let $i \geq 2, n \geq 0, \delta:=\delta_{i}(n)$. Then we have

a) If $i>1, \sum_{t=0}^{r}\left(n-n_{\langle i\rangle}\right)_{\langle i-1\rangle(t)}=n$, for every $r \geq \delta$

b) Let $j=1$ and $r>\delta$; then

$$
\sum_{t=0}^{r}\left(n-n_{\langle i\rangle}\right)_{\langle i-1\rangle(t)}=n+n(2)-n(1) .
$$


c) Let $j=1$ and $p$ the maximum integer such that $n(p)=n(2)+p$ -2 ; then

$$
\sum_{t=0}^{\delta}\left(n-n_{\langle i\rangle}\right)_{\langle i-1\rangle(t)}= \begin{cases}n+n(2)-n(1) & \text { if } p<i \\ n+n(2)-n(1)-1 & \text { if } p=i .\end{cases}
$$

d) If $n$ is i-regular then

$$
\sum_{t=0}^{\delta}\left(n-n_{\langle i\rangle}\right)_{\langle i-1\rangle(t)}=n .
$$

e) For every $r \geq \delta$ we have

$$
\sum_{t=0}^{r}\left(n-n_{\langle i\rangle}\right)_{\langle i-1\rangle(t)} \geq n .
$$

Proof. If $j>1$, it is clear that $\delta=\delta_{i-1}\left(n-n_{\langle i\rangle}\right)$, hence

$$
\sum_{i=0}^{r}\left(n-n_{\langle i\rangle}\right)_{\langle i-1\rangle(t)}=\left(n-n_{\langle i\rangle}\right)^{\langle i-1\rangle}=n
$$

where the first equality follows by Property 1.12 and the second by Property 1.7. This proves a).

Let $j=1$ and $r>\delta$. It is clear that $\delta_{i-1}\left(n-n_{\langle i\rangle}\right) \leq \delta+1$, hence we get

$$
\sum_{t=0}^{r}\left(n-n_{\langle i\rangle}\right)_{\langle i-1\rangle(t)}=\left(n-n_{\langle i\rangle}\right)^{\langle i-1\rangle}=n+n(2)-n(1)
$$

again by the same properties. This proves b).

Let $j=1$ and $p<i$. Then it is clear that $\delta_{i-1}\left(n-n_{\langle i\rangle}\right)=\delta$, hence

$$
\sum_{i=0}^{\delta}\left(n-n_{\langle i\rangle}\right)_{\langle i-1\rangle(t)}=\left(n-n_{\langle i\rangle}\right)^{\langle i-1\rangle}=n+n(2)-n(1) .
$$

This proves the first case of $c$ ).

Now let $j=1$ and $p=i$. Then $n_{-1}=\left(\begin{array}{c}n(2)+i-2 \\ i-1\end{array}\right)$ by Property 1.11 . Hence $\delta_{i-1}\left(n_{-1}\right)=n(2)-1$ and $\delta=n(i)-i=n(2)+i-2-i=n(2)-2$, hence

$$
\begin{aligned}
\sum_{t=0}^{\delta}\left(n_{-1}\right)_{\langle i-1\rangle(t)} & =\left(n_{-1}\right)^{\langle i-1\rangle}-\left(n_{-1}\right)_{\langle i-1\rangle(n(2)-1)} \\
& =n+n(2)-n(1)-\left(\begin{array}{c}
n(2)+i-2-n(2)+1 \\
i-1
\end{array}\right) \\
& =n+n(2)-n(1)-1 .
\end{aligned}
$$

This proves the second case of $c$ ). 
Finally d) and e) are easy consequences of the previous assertions.

\section{§ 2. Theorems of Macaulay, Stanley, Green and lex-segments}

Let $A$ be a standard $k$-algebra i.e. $A=R / I=k\left[x_{1}, \cdots, x_{h}\right] / I$ where $\operatorname{deg}\left(x_{i}\right)=1$ for $i=1, \cdots, h$ and $I$ is a homogeneous ideal. As usual $H_{A}(n)$ denotes the dimension of the $k$-vectorspace $A_{n}$ of elements of degree $n$ of $A$ and $H_{A}$ is called the Hilbert function of $A$. Let $T_{R}$ be the monoid of terms in $x_{1}, \cdots, x_{n}$ i.e. $T_{R}:=\left\{\left(x_{1}^{a_{1}} \cdots x_{h}^{a_{h}} / a_{i} \in \mathbb{N}\right\}\right.$ and let deglex (short from for degree-lexicographic ordering) denote the total ordering on $T_{R}$ defined by the following rule: $x_{1}^{a_{1}} \ldots x_{h}^{a_{h}}>x_{1}^{b_{1}} \ldots x_{h}^{b_{h}}$ if the first non zero (from the left) coordinate of $\left(\sum_{i}\left(a_{i}-b_{i}\right), a_{1}-b_{1}, \cdots, a_{h}-b_{h}\right)$ is positive. For every $n$, the terms of degree $n$ of $R$ are totally ordered by lex. For instance if $h=3$ and $n=2$ we get $x_{1}^{2}>x_{1} x_{2}>x_{1} x_{3}>x_{2}^{2}>x_{2} x_{3}>x_{3}^{2}$. It makes therefore sense to talk about lex-segments. In the following we only consider segments of terms in $R_{n}$ starting from the first one i.e. $x_{1}^{n}$; for instance $x_{1}^{2}, x_{1} x_{2}, x_{1} x_{3}$ is a lex-segment.

Definition. Let $I=\oplus I_{n}$ be a graded ideal of $k\left[x_{1}, \cdots, x_{h}\right]$. We say that $I$ is a lex-segment ideal if $I_{n}$ is generated as a $k$-vectorspace by a lex-segment of terms for every $n$.

Let us now recall the following theorems of Macaulay, Stanley and Green.

TheOREm 2.1. Let $V$ be a k-subvectorspace of $R_{a}$ and let $W$ be the $k$-subvectorspace of $R_{d}$ generated by the lex-segment of length $\operatorname{dim} V$. Then

a) $R_{1} W$ is generated by a lex-segment

b) $\operatorname{dim}\left(R_{1} W\right) \leq \operatorname{dim}\left(R_{1} V\right)$.

Proof. See Macaulay (1927)

THeOREM 2.2. Let $H: \mathbb{N} \rightarrow \mathbb{N}$ be a function and let $k$ be any field. The following conditions are equivalent

a) There exists a standard k-algebra $A$ with $A_{0}=k$ and with Hilbert function $H$.

b) There exists a lex-segment ideal $J$ such that $H=H_{R / J}$.

c) $H(0)=1$ and for $n \geq 1 H(n+1) \leq H(n)^{\langle n\rangle}$.

Proof. See Stanley (1978)

Theorem 2.3. Let $V$ be a $k$-subvectorspace of $R_{d}$ of codimension $c$. 
Let $H$ be a general hyperplane, $V_{H}:=(V+H) / H$ i.e. the restriction of $V$ to $H$, and $c_{H}$ the codimension of $V_{H}$ in $(R / H)_{d}$. Then $c_{H} \leq c_{\langle d\rangle}$.

Proof. See Green (1988)

THEOREM 2.4. Let $W$ be a $k$-subvectorspace of $R_{d}$ of codimension $c$, which is a lex-segment. Then

a) $\operatorname{codim}\left(R_{1} W\right)=c^{\langle d\rangle}$.

Let $H$ be a general hyperplane, $W_{H}$ the restriction of $W$ to $H$, and $c_{H}$ the codimension of $W_{H}$, then

b) $c_{H}=c_{\langle d\rangle}$.

Proof. These facts are generally attributed to Stanley (1978). An easy proof can be obtained by suitably using the arguments of Green (1988)

Remark. A full detailed proof of the previous theorems will appear in (Robbiano-Valla).

Proposition 2.5. Let $W$ be a k-subvectorspace of $R_{d}$ of codimension $c$, which is a lex-segment, $H=x_{h}, W_{H}$ the restriction of $W$ to $H$, and $c_{H}$ the codimension of $W_{H}$. Then $c_{H}=c_{\langle d\rangle}$.

Proof. Let $L:=a_{1} x_{1}+\cdots+a_{h} x_{h}$ be an hyperplane satisfying b) of Theorem 2.4 and let $f$ be the linear automorphism of $R$ given by $f\left(x_{i}\right)=x_{i}$ for $i=1, \cdots, h-1, f\left(x_{h}\right)=a_{1} x_{1}+\cdots+a_{h} x_{h}$. Then $f^{-1}(L)=x_{h}$, hence it suffices to show that it fixes $W$. Let $T=x_{1}^{a_{1}} \cdots x_{h}^{a_{h}}$ be a term in $W$; if $a_{h}=0$ then $f^{-1}(T)=T$, otherwise $f^{-1}(T)$ is a sum of monomials, whose associated terms are bigger than or equal to $T$, hence they are in $W$ since $W$ is a lex-segment. We get $f^{-1}(W) \subseteq W$, hence they coincide since they have the same codimension

Corollary 2.6. Let $I$ be a homogeneous ideal of $R, A=R / I$. Then

a) $\operatorname{dim}\left(I_{n+1} / I_{n} \cdot R_{1}\right) \leq H_{A}(n)^{\langle n\rangle}-H_{A}(n+1)$ for every $n$.

b) $\operatorname{dim}\left(R_{n+1}\right)-\operatorname{dim}\left(I_{n} \cdot R_{1}\right) \leq H_{A}(n)^{\langle n\rangle}$ for every $n$.

c) $H_{A}(n)^{\langle n\rangle}=H_{A}(n+1)$ for $n \gg 0$.

Proof. It is clear that a) and b) are equivalent, and b) is nothing but Theorem $2.2 \mathrm{c}$ ) applied to the ideal generated by $I_{n}$. c) Let $J$ be the lex-segment ideal with the same Hilbert function of $I$ and let $\delta$ be the maximal degree of a minimal set of generators of $J$. If $n>\delta$ then 
of course $J_{n} \cdot R_{1}=J_{n+1}$ hence

$$
H_{A}(n)^{\langle n\rangle}=\operatorname{dim}\left(R_{n+1}\right)-\operatorname{dim}\left(J_{n+1}\right) \quad \text { by } 2.4 \text { a) }
$$

and

$$
\operatorname{dim}\left(R_{n+1}\right)-\operatorname{dim}\left(J_{n+1}\right)=H_{R / J}(n+1)=H_{A}(n+1) .
$$

Corollary 2.7. Let $I$ be a homogeneous ideal of $R$, and $J$ the corresponding lex-segment ideal. Then

a) $\nu(I)=\sum_{1}^{\infty} n \operatorname{dim}\left(I_{n+1} / I_{n} \cdot R_{i}\right)$

b) $\nu(I) \leq \nu(J)$

c) $\nu(J)=\sum_{1}^{\infty}{ }_{n}\left(H_{A}(n)^{\langle n\rangle}-H_{A}(n+1)\right)$.

Proof. a) is obvious

b) $\nu(I)=\sum_{1}^{\infty} \operatorname{dim}\left(I_{n+1} / I_{n} \cdot R\right)=\sum_{1}^{\infty}\left(\operatorname{dim}\left(I_{n+1}\right)-\operatorname{dim}\left(I_{n} \cdot R_{1}\right)\right)$

$$
=\sum_{1}^{\infty}{ }_{n}\left(\operatorname{dim}\left(J_{n+1}\right)-\operatorname{dim}\left(I_{n} \cdot R_{1}\right)\right) \leq \sum_{1}^{\infty}{ }_{n}\left(\operatorname{dim}\left(J_{n+1}\right)-\operatorname{dim}\left(J_{n} \cdot R_{1}\right)\right)
$$

and $\quad \sum_{1}^{\infty} n\left(\operatorname{dim}\left(J_{n+1}\right)-\operatorname{dim}\left(J_{n} \cdot R_{1}\right)\right)=\nu(J) \quad$ by a $)$

c) $\nu(J)=\sum_{1}^{\infty} \operatorname{dim}\left(J_{n+1} / J_{n} \cdot R_{1}\right) \quad$ by a)

hence

$$
\nu(J)=\sum_{1}^{\infty}\left(\operatorname{dim}\left(J_{n+1}\right)-\operatorname{dim}\left(R_{n+1}\right)+H_{A}(n)^{\langle n\rangle}\right) \quad \text { by } 2.4 \text { a) }
$$

and the conclusion follows

Definition. Given a monomial ideal $I$ in $R=k\left[x_{1}, \cdots, x_{h}\right]$, we denote by $I_{\langle r\rangle}$ the image of the ideal $I$ in $R_{\langle r\rangle}:=k\left[x_{1}, \cdots, x_{r-1}\right]$ under the canonical projection. It is clear that if $I$ is a lex-segment, then $I_{\langle r\rangle}$ is a lexsegment too.

Definition. Given a homogeneous ideal $I$ in $R=k\left[x_{1}, \cdots, x_{h}\right]$, we denote by $e(R / I)$ the multiplicity of $R / I$, i.e. the degree of the associated projective scheme. We denote by indeg $(I)$ or indeg $(R / I)$ the minimum $s$ such that $I_{s} \neq 0$.

Corollary 2.8. Let $J$ be a lex-segment ideal.

Then $H_{R_{\langle n\rangle} / J_{\langle h\rangle}}(n)=H_{A}(n)_{\langle n\rangle}$ for every $n \geq 1$.

Proof. It follows immediately from Proposition 2.5 
Theorem 2.9. Let $J$ be a zero-dimensional lex-segment ideal with initial degree bigger than one. Then

$$
\nu(J)=\nu\left(J_{\langle h\rangle}\right)+e\left(R_{\langle h\rangle} / J_{\langle h\rangle}\right) .
$$

Proof. We have already remarked that $J_{\langle h\rangle}$ is a lex-segment, hence we get

$$
\begin{aligned}
& \left.\nu\left(J_{\langle h\rangle}\right)=\sum_{1}^{\infty}{ }_{n}\left(H_{R_{\langle h\rangle} / J_{\langle h\rangle}}(n)^{\langle n\rangle}-H_{R_{\langle h\rangle} / J_{\langle h\rangle}}(n+1)\right) \quad \text { by } 2.7 \mathrm{c}\right) . \\
& \left.=\sum_{1}^{\infty}\left(H_{A}(n)_{\langle n\rangle}\right)^{\langle n\rangle}-H_{R_{\langle h\rangle} / J_{\langle h\rangle}}(n+1)\right) \quad \text { by } 2.8 \\
& =\sum_{1}^{\infty}{ }_{n}\left(H_{A}(n)^{\langle n\rangle}-H_{A}(n)-H_{R_{\langle n\rangle} / J}(n\rangle=1\right) \text { by Property } 1.6 .
\end{aligned}
$$

Cleary

$$
e\left(R_{\langle h\rangle} / J_{\langle h\rangle}\right)=\sum_{1}^{\infty} H_{R_{\langle h\rangle} / J_{\langle h\rangle}}(n)+1
$$

Theorefore

$$
\begin{aligned}
\nu\left(J_{\langle h\rangle}\right) & +e\left(R_{\langle h\rangle} / J_{\langle h\rangle}\right) \\
& =\sum_{1}^{\infty}{ }_{n}\left(H_{A}(n)^{\langle n\rangle}-H_{A}(n)-H_{R_{\langle h\rangle} / J_{\langle h\rangle}}(n+1)+H_{R_{\langle h\rangle} / J}(n)\right)+1 \\
& =\sum_{1}^{\infty}\left(H_{A}(n)^{\langle n\rangle}-H_{A}(n)\right)+H_{R_{\langle h\rangle} / J\langle h\rangle}(1)+1 \\
& =\sum_{1}^{\infty}\left(H_{A}(n)^{\langle n\rangle}-H_{A}(n+1)\right)-H_{A}(1)+H_{R_{\langle h\rangle} / J\langle h\rangle}(1)+1 \\
& =\sum_{1}^{\infty}{ }_{n}\left(H_{A}(n)^{\langle n\rangle}-H_{A}(n+1)\right) \\
& \quad \text { since the initial degree of } J \text { is bigger than one. } \\
& =\nu(J) \text { by 2.7.c. }
\end{aligned}
$$

Corollary 2.10. Let $J$ be a zero-dimensional lex-segment ideal with initial degree bigger than one. Then

$$
\nu(J)=\sum_{1}^{h} e\left(R_{\langle i\rangle} / J_{\langle i\rangle}\right)
$$

Proof. We remark that $J_{\langle i\rangle}$ is a lex-segment ideal with the same initial degree as $J$ for $i>1$, and $J_{\langle 1\rangle}=0$ hence we can apply repeatedly Theorem 2.9 and the conclusion follows 


\section{§ 3. Main tools}

We recall the following well-known notion, which is motivated by Theorem $2.2 \mathrm{c})$.

Definition. Let $H: \mathbb{N} \rightarrow \mathbb{N}$ be a function. We call it an $O$-sequence if $H(0)=1$ and for all $n \geq 1 H(n+1) \leq H(n)^{\langle n\rangle}$. We call it a zerosequence if it is an $O$-sequence and $H(n)=0$ for large $n$.

Theorem $2.2 \mathrm{c}$ ) says that $O$-sequences are those sequences which occur as Hilbert functions of standard $k$-algebras and of course zerosequences are those which occur as Hilbert functions of zero-dimensional standard $k$-algebras.

We observe that if $H$ is an $O$-sequences and $H(n)=0$, then $H(m)=0$ for every $m \geq n$, hence zero-sequences can be represented by sequences of type $(H(1), H(2), \cdots, H(n-1), 0)$.

Definition. Given an $O$-sequence $H \neq(1,0)$, we denote by indeg $(H):=$ $\min \left(n / H(n) \neq\left(\begin{array}{c}H(1)+n-1 \\ n\end{array}\right)\right)$ and we call jt the initial degree of $H$.

We remark that indeg $(H)$ is either $\infty$ or a natural number bigger than 1 ; moreover if $H$ is a zero-sequence, then either $H=(1,0)$ or indeg $(H)$ $<\infty$.

Definition. Given a zero-sequence $H$, we denote by $\operatorname{socdeg}(H):=$ $\max (n / H(n) \neq 0)$.

Lemma 3.1. For every zero-sequence $H \neq(1.0)$, indeg $(H) \leq \operatorname{socdeg}(H)$ +1 .

Proof. Let $s=: \operatorname{socdeg}(H)$. Then $H(1) \geq 1$ and $H(s+1)=0 \neq$ $\left(\begin{array}{c}H(1)+s \\ s+1\end{array}\right)$

Definition. Given an $O$-sequence $H$, we denote by $H_{\prec 〉}$ the sequence defined by

$$
H_{\langle\rangle}(0):=1, \quad H_{\langle\rangle}(n):=H(n)_{\langle n\rangle} \quad \text { if } n \geq 1 ;
$$

inductively we define $H_{\langle\rangle(r)}=\left(H_{\langle\rangle(r-1)}\right)_{\langle\rangle}$. We denote by $H_{-1}$ the sequence defined by

$$
\begin{aligned}
& H_{-1}:=(1,0) \quad \text { if } H=(1,0), \\
& H_{-1}(n):=H(n+1)-H(n+1)_{\langle n+1\rangle}=H(n+1)_{-1}
\end{aligned}
$$


for every $n \geq 0$ if $H \neq(1,0)$. We remark that we use the symbol $H(n+1)_{-1}$ instead of $H(n+1)(n+1)_{-1}$ for the sake of simplicity.

Proposition 3.2. a) If $H$ is an $O$-sequence, then $H_{\zeta>}$ is an $O$-sequence.

b) If $H$ is an O-sequence, then $H_{-1}$ is an O-sequence.

Proof. a) We need to show that

$$
H(n+1)_{\langle n+1\rangle} \leq\left(H(n)_{\langle n\rangle}\right)^{\langle n\rangle} \quad \text { for } n \geq 1 .
$$

But

$$
H(n+1)_{\langle n+1\rangle} \leq\left(H(n)^{\langle n\rangle}\right)_{\langle n+1\rangle} \quad \text { by Property } 1.3
$$

and

$$
\left(H(n)^{\langle n\rangle}\right)_{\langle n+1\rangle}=\left(H(n)_{\langle n\rangle}\right)^{\langle n\rangle} \quad \text { by Property } 1.6
$$

b) If $H=(1,0)$, there is nothing to be proved. Let $H \neq(1,0)$; we need to show that

$$
H(n+2)_{-1} \leq\left(H(n+1)_{-1}\right)^{\langle n\rangle} \quad \text { for } n \geq 1 .
$$

But

$$
H(n+2)_{-1} \leq H(n+1) \quad \text { by Property } 1.8
$$

and

$$
H(n+1) \leq\left(H(n+1)-H(n+1)_{\langle n+1\rangle}\right)^{\langle n\rangle} \quad \text { by Property } 1.7
$$

Definition. Let $H$ be a zero-sequence. We say that $H$ is a TVSsequence (Three Values Suffice) if either

$$
\begin{aligned}
& H=(1,0) \quad \text { or } \\
& \text { indeg }(H) \geq \operatorname{socdeg}(H)-1 \text { or } \\
& H(n+1)=H(n)^{\langle n\rangle} \text { for every } n=\operatorname{indedg}(H), \cdots, \operatorname{socdeg}(H)-2 .
\end{aligned}
$$

The reason for the name is that by its very definition a TVS-sequence is determined by $H(1), H(i), H(s)$, where $i=\operatorname{indeg}(H), s=\operatorname{socdeg}(H)$.

Definition. Let $H$ be a TVS-sequence. We say that $H$ is a special sequence if either

$$
\begin{aligned}
& H=(1,0) \text { or } \\
& \text { indeg }(H) \geq \operatorname{socdeg}(H) \text { or } \\
& H(i)=\left(\begin{array}{c}
H(1)+i-2 \\
i
\end{array}\right)+\cdots+\left(\begin{array}{c}
H(1)+j-2 \\
j
\end{array}\right) \text { where } i=\operatorname{indeg}(H) .
\end{aligned}
$$

We observe that this is a strong condition of $i$-regularity for $H(i)$. 
Examples. 1) $H=(1,3,6,0)$ is special since $3=\operatorname{indeg}(H)=$ socdeg $(H)+1$

2) $H=(1,3,4,0)$ is special since $2=\operatorname{indeg}(H)=\operatorname{socdeg}(H)$

3) $H=(1,3,6,9,12,7,0)$ is special since $3=\operatorname{indeg}(H), \operatorname{socdeg}(H)$ $=5$, $H(3)=\left(\begin{array}{c}3+3-2 \\ 3\end{array}\right)+\left(\begin{array}{c}3+2-2 \\ 2\end{array}\right)+\left(\begin{array}{c}3+1-2 \\ 1\end{array}\right), H(4)=H(3)^{\langle 3\rangle}$

4) $H=(1,3,4,1,0)$ is a TVS-sequence, but it is not special since $4=H(2)$ is not of the prescribed type

5) $H(1,3,5,6,2,0)$ is not a TVS-sequence, since $6=H(3) \neq H(2)^{\langle 2\rangle}$ $=7$

6) $H=(1,3,1,1,0)$ is a TVS-sequence, but it is not special since $1=H(2)<\left(\begin{array}{c}H(1)+i-2 \\ i\end{array}\right)=3$.

LEMma 3.3. Let $H$ be a zero-sequence. Then

a) $H_{\langle\rangle}=(1,0)$ or indeg $\left(H_{\langle\rangle}\right)=\operatorname{indeg}(H)$

b) $\operatorname{socdeg}\left(H_{\langle>}\right) \leq \operatorname{socdeg}(H)$

c) $H_{-1}=(1,0)$ or indeg $\left(H_{-1}\right) \geq \operatorname{indeg}(H)-1$

d) $H=(1,0)$ or $\operatorname{socdeg}\left(H_{-1}\right)=\operatorname{socdeg}(H)-1$.

Proof. a) and b) are clear.

c) If $H_{-1} \neq(1,0)$ then indeg $\left(H_{-1}\right) \geq 2$ hence the result holds trivially if indeg $(H) \leq 3$. Now let indeg $(H) \geq 4$; then

$$
H(2)=\left(\begin{array}{c}
H(1)+2-1 \\
2
\end{array}\right) \text { hence } H_{-1}(1)=H(2)_{-1}=\left(\begin{array}{c}
H(1)+1-1 \\
1
\end{array}\right)=H(1) .
$$

It suffices to show that $H_{-1}(n)=\left(\begin{array}{c}H(1)+n-1 \\ n\end{array}\right)$ for every $n \leq \operatorname{indeg}(H)$ -2. And indeed $H_{-1}(n)=H(n+1)_{-1}=\left(\begin{array}{c}H(1)+n-1 \\ n\end{array}\right)$ since $n+1 \leq$ $\operatorname{indeg}(H)-1$.

d) If $H \neq(1,0)$, then the conclusion follows from the obvious fact that $c_{-1} \neq 0$ if $c \neq 0$.

Proposition 3.4. a) If $H$ is a TVS-sequence, then $H_{\zeta\rangle}$ is a TVSsequence.

b) If $H$ is a special sequence, then $H_{\langle 〉}$ is a special sequence.

Proof. a) If $H=(1,0)$, there is nothing to be proved.

Now either $H_{\langle\rangle}=(1,0)$ or $\operatorname{indeg}\left(H_{\langle\rangle}\right)=\operatorname{indeg}(H)$; we explicitly remark that for example $H_{\langle\rangle}=(1,0)$ holds for $H=(1,1,1,1,0)$. Moreover 
$\operatorname{socdeg}\left(H_{\langle\rangle}\right) \leq \operatorname{socdeg}(H)$ hence $\operatorname{indeg}(H) \geq \operatorname{socdeg}(H)-1$ is preserved. Finally let $H(n+1)=H(n)^{\langle n\rangle}$ for every $n=\operatorname{indeg}(H), \cdots, \operatorname{socdeg}(H)-2$. Then

$$
H_{\langle\rangle}(n+1)=H(n+1)_{\langle n+1\rangle}=\left(H(n)^{\langle n\rangle}\right)_{\langle n+1\rangle}=\left(H(n)_{\langle n\rangle}\right)^{\langle n\rangle}=H_{\langle\rangle}(n)^{\langle n\rangle}
$$

and the conclusion follows.

b) We have

$$
H_{\langle\rangle}(i)=H(i)_{\langle i\rangle}=\left(\begin{array}{c}
H(1)-1+i-2 \\
i
\end{array}\right)+\cdots+\left(\begin{array}{c}
H(1)-\underset{j}{1}-j-2 \\
j
\end{array}\right)
$$

where $i=\operatorname{indeg}(H)$. The conclusion follows since $H_{\langle\rangle}(1)=H(1)-1$ and indeg $\left(H_{\iota 〉}\right)=\operatorname{indeg}(H)$

It is not true that if $H$ is a TVS-sequence, then $H_{-1}$ is a TVSsequence as the following example shows.

Example. $\quad H=(1,3,6,3,3,1,0) ; H_{-1}=(1,3,3,1,0)$ which is not a TVS-sequence since indeg $\left(H_{-1}\right)=2$, socdeg $\left(H_{-1}\right)=4,1=H_{-1}(3) \neq H_{-1}(2)^{\langle 2\rangle}$. However we have the following

Proposition 3.5. If $H$ is a special sequence, then $H_{-1}$ is a special sequence.

Proof. If $H=(1,0)$ there is nothing to prove.

Let now $i:=\operatorname{indeg}(H)$, and $s:=\operatorname{socdeg}(H)$. Let $i \geq s$; if $H_{-1}=(1,0)$ there is nothing to prove. Otherwise by Lemma $3.3 \mathrm{c})$ we have indeg $\left(H_{-1}\right)$ $\geq i-1 \geq s-1=\operatorname{socdeg}\left(H_{-1}\right)$ by Lemma $\left.3.3 \mathrm{~d}\right)$ and the conclusion follows.

So now we may asume that $2 \leq i<s$ hence $H(i)=\left(\begin{array}{c}H(1)+i-2 \\ i\end{array}\right)$ $+\cdots+\left(\begin{array}{c}H(1)+j-2 \\ j\end{array}\right)$ where $i=\operatorname{indeg}(H)$, by definition of special zerosequence.

ClaIm. Under this assumption $H(n)=H_{-1}(n)$ for $n=0, \cdots, i-2$ and for $n=i, \cdots, s-2$.

Namely $H_{-1}(n)=H(n+1)_{-1}=\left(H(n)^{\langle n\rangle}\right)_{-1}$ since $H$ is TVS and $\left(H(n)^{\langle n\rangle}\right)_{-1}$ $=H(n)$. This concludes the proof of the Claim.

We now consider three different cases;

Case 1). $j=1$.

Then

$$
H_{-1}(i-1)=H(i)_{-1}=\sum_{0}^{i-1} r\left(\begin{array}{c}
H(1)+r-2 \\
r
\end{array}\right)=\left(\begin{array}{c}
H(1)+i-2 \\
i-1
\end{array}\right)=H(i-1)
$$


hence $H(n)=H_{-1}(n)$ for $n=0, \cdots, s-2$. But $s-2=\operatorname{socdeg}\left(H_{-1}\right)-1$ by Lemma $3.3 \mathrm{~d}$ ) and the conclusion follows.

Case 2). $\quad 2<i$ and $j>1$.

We show that in this case indeg $\left(H_{-1}\right)=i-1$. Namely $H_{-1}(n)=H(n)$ for $n \leq i-2$; in particular $H_{-1}(1)=H(1)$. Furthermore

$$
\begin{aligned}
H_{-1}(i-1)=H(i)_{-1} & =\left(\begin{array}{c}
H(1)+i-3 \\
i-1
\end{array}\right)+\cdots+\left(\begin{array}{c}
H(1)+j-3 \\
j-1
\end{array}\right) \\
& <\left(\begin{array}{c}
H(1)+i-2 \\
i-1
\end{array}\right)
\end{aligned}
$$

whence indeg $\left(H_{-1}\right)=i-1$. To show that $H_{-1}$ is a TVS-sequence we only need to prove that $H_{-1}(i)=H_{-1}(i-1)^{\langle i-1\rangle}$. Indeed $H_{-1}(i)=H(i)$ by the Claim, and

$$
H_{-1}(i-1)^{\langle i-1\rangle}=\left(H(i)_{-1}\right)^{\langle i-1\rangle}=H(i) \quad \text { by Property } 1.7 .
$$

Finally, since $H_{-1}(1)=H(1)$ and

$$
H_{-1}(i-1)=H(i)_{-1}=\left(\begin{array}{c}
H(1)+i-3 \\
i-1
\end{array}\right)+\cdots+\left(\begin{array}{c}
H(1)+j-3 \\
j-1
\end{array}\right),
$$

we get the required formula for the speciality of $H_{-1}$

Case 3). $\quad i=2$ and $j>1$ hence $j=2$.

We may assume $H(1)>1$ otherwise $H_{-1}=(1,0)$. We get $H(2)=$ $\left(\begin{array}{c}H(1) \\ 2\end{array}\right)$ hence $H_{-1}(1)=H(2)_{-1}=H(1)-1>0$. By the Claim we get $H_{-1}(n)$ $=H(n)$ for $n=2, \cdots, s-2$. Since $H$ is a TVS-sequence and $H(2)=$ $\left(\begin{array}{c}H(1) \\ 2\end{array}\right)$, it follows that

$$
H_{-1}(n)=\left(\begin{array}{c}
H(1)+n-2 \\
n
\end{array}\right)=\left(\begin{array}{c}
H(1)-1+n-1 \\
n
\end{array}\right) .
$$

This proves that indeg $\left(H_{-1}\right) \geq s-1=\operatorname{socdeg}\left(H_{-1}\right)$ and the conclusion follows

Definition. Given a zero-sequence $H$, we denote by $e(H):=\sum_{0}^{\infty} i(i)$ and we call it the multiplicity of $H$. We remark that $e(H) \geq 1$ for every $H$ and equality holds iff $H=(1,0)$.

Lemma 3.6. Let $H$ be a zero-sequence, $H \neq(1,0)$. Then $e(H)=$ $e\left(H_{\langle\rangle}\right)+e\left(H_{-1}\right)$. 
Proof. $\quad e\left(H_{\langle\rangle}\right)=\sum_{0}^{\infty} H(i)_{\langle i\rangle}$;

$$
e\left(H_{-1}\right)=\sum_{0}^{\infty} H_{-1}(i)=\sum_{0}^{\infty} H(i+1)_{-1}=\sum_{1}^{\infty} H(i)_{-1} .
$$

Hence

$$
e\left(H_{\langle\rangle}\right)+e\left(H_{-1}\right)=1+\sum_{1}^{\infty}\left(H(i)_{\langle i\rangle}+H(i)_{-1}\right)=1+\sum_{1}^{\infty}{ }_{i} H(i)=e(H)
$$

Lemma 3.7. Let $H$ be a zero-sequence. Then $\delta_{n}(H(n)) \geq \delta_{n+1}(H(n+1))$ for every $n \geq 0$.

Proof. It is an easy consequence of Theorem $2.2 \mathrm{c}$ ) and Property 1.1

Definition. Let $H, K$ be zero-sequences. We write $H \cdot \geq K$ if $H(n)$ $\geq K(n)$ for every $n=0, \cdots, \operatorname{socdeg}(H)-1$.

Lemma 3.8. Let $H, K$ be zero-sequences. If $H \cdot \geq K$, then $H_{-1} \cdot \geq K_{-1}$ and $H_{\iota 〉} \cdot \geq K_{\langle\rangle}$.

Proof. The proof easily follows from Property 1.5 and Property 1.3

MaIN Lemma 3.9. Let $H, K$ be zero-sequences and assume that $H$ be special. Let $i:=\operatorname{indeg}(H), s:=\operatorname{socdeg}(H)$ and if $s>0$ let

$$
H(s):=\left(\begin{array}{c}
n(s) \\
s
\end{array}\right)+\left(\begin{array}{c}
n(s-1) \\
s-1
\end{array}\right)+\cdots+\left(\begin{array}{c}
n(j) \\
j
\end{array}\right)
$$

be the s-expansion of $H(s)$. If $H \cdot \geq K$ and $e(H) \geq e(K)$ then

a) $e\left(H_{\langle\rangle}\right) \geq e\left(K_{\langle\rangle}\right)$

b) If moreover $n(j)>j$ and $e(H)>e(K)$ then $e\left(H_{\langle 〉}\right)>e\left(K_{\langle\rangle}\right)$.

Proof. It is enough to prove a). For, we may assume $s>0$, otherwise b) is empty. So let $n(j)>j$ and $e(H)>e(K)$. We have

$$
\begin{aligned}
H(s)-1= & \left(\begin{array}{c}
n(s) \\
s
\end{array}\right)+\left(\begin{array}{c}
n(s-1) \\
s-1
\end{array}\right)+\cdots+\left(\begin{array}{c}
n(j) \\
j
\end{array}\right)-1 \\
= & \left(\begin{array}{c}
n(s) \\
s
\end{array}\right)+\left(\begin{array}{c}
n(s-1) \\
s-1
\end{array}\right)+\cdots+\left(\begin{array}{c}
n(j)+1 \\
j+1
\end{array}\right) \\
& +\left(\begin{array}{c}
n(j)-1 \\
j
\end{array}\right)+\cdots+\left(\begin{array}{c}
n(j)-j \\
1
\end{array}\right)
\end{aligned}
$$

hence $(H(s)-1)_{\langle s\rangle}=(H(s))_{\langle s\rangle}-1$.

Let $H^{\prime}:=(1, H(1), \cdots, H(s-1), H(s)-1,0) ;$ then $e(H)=e\left(H^{\prime}\right)+1$ 
hence $e\left(H^{\prime}\right) \geq e(K)$. It is easy to check that $H^{\prime}$ is special and $H^{\prime} \cdot \geq K$. By a) we get $e\left(H_{\langle\rangle}^{\prime}\right) \geq e\left(K_{\langle\rangle}\right)$and from $(H(s)-1)_{\langle s\rangle}=(H(s))_{\langle s\rangle}-1$ we get $e\left(H^{\prime}\right)_{\langle\rangle}=e\left(H_{\langle\rangle}\right)-1$.

We now prove the theorem by induction on $s$. If $s=0$ i.e. $H=(1,0)$, the conclusion is trivial. Let $s>0$. If $K=(1,0)$ there is nothing to prove. If $K \neq(1,0)$ we have $e(H)=e\left(H_{\zeta\rangle}\right)+e\left(H_{-1}\right)$ and $e(K)=e\left(K_{\langle\rangle}\right)+$ $e\left(K_{-1}\right)$ by Lemma 3.6. Let $\varepsilon:=e(H)-e(K)$; then $\varepsilon \geq 0$ and a) is equivalent to

$$
e\left(H_{-1}\right) \leq \varepsilon+e\left(K_{-1}\right) .
$$

Let us assume by contradiction that

$$
e\left(H_{-1}\right)>\varepsilon+e\left(K_{-1}\right) .
$$

By Proposition 3.5 we have that $H_{-1}$ is special. By Lemma 3.8 we get $H_{-1} \cdot \geq K_{-1}$ and $\left(H_{-1}\right)_{\langle\rangle(r)} \cdot \geq\left(K_{-1}\right)_{\langle\rangle(r)}$ for every $r \geq 0$. By Lemma 3.3 socdeg $\left(H_{-1}\right)=s-1$; by Proposition 3.4 the $O$-sequences $\left(H_{-1}\right)_{\langle\rangle(r)}$ are special, hence we can apply induction and we get

$$
e\left(\left(H_{-1}\right)_{\langle>(r)}\right) \geq e\left(\left(K_{-1}\right)_{\langle>(r)}\right) \quad \text { for every } r \geq 1 .
$$

If $d$ is any natural number, we sum (1) and (2) for $r=1$ to $d$ and we get

$$
\sum_{0}^{d} e\left(\left(H_{-1}\right)_{\langle>(r)}\right)>\varepsilon+\sum_{0}^{d} e\left(\left(K_{-1}\right)_{\langle>(r)}\right) .
$$

By definition

$$
\left(H_{-1}\right)_{\langle\rangle(r)}=\left(1,\left(H(2)_{-1}\right)_{\langle 1\rangle(r)}, \cdots,\left(H(s)_{-1}\right)_{\langle s-1\rangle(r)}, 0\right)
$$

hence

$$
e\left(\left(H_{-1}\right)_{\langle>(r)}=1+\sum_{1}^{s-1}\left(H(n+1)_{-1}\right)_{\langle n\rangle(r)}\right)
$$

and the like for $\left(K_{-1}\right)_{\langle\rangle(r)}$. If $t$ denotes socdeg $(K)$ then (3) can be rewritten as

$$
\begin{aligned}
1+d+ & \sum_{1}^{s-1} n\left(\sum_{0}^{d}\left(H(n+1)_{-1}\right)_{\langle n\rangle(r)}\right) \\
& >\varepsilon+1+d+\sum_{1}^{t-1} n\left(\sum_{1}^{d}\left(K(n+1)_{-1}\right)_{\langle n\rangle(r)}\right) .
\end{aligned}
$$

We treat separately two different cases;

Case 1. $i>s$ 
By Lemma 3.1 we get $i=s+1$ and this means that $H(i)=$ $\left(\begin{array}{c}H(1)+i-1 \\ i\end{array}\right)$ for $i=0, \cdots, s$ and $H(i)=0$ for $i>s$.

Let $d:=\delta_{1}(H(1))=H(1)-1$; If $s=1$ then $H(1) \geq K(1)$ since $e(H) \geq$ $e(K)$; If $s>1$, then $H(1) \geq K(1)$ since $H \cdot>K$. In any case it is clear that $d=\delta_{n}(H(n))$ for $n=1, \cdots, s$ and $d \geq \delta_{n}(K(n))$ for $n=1, \cdots, t$ by Property 1.1 and Lemma 3.7.

Now use (4) and Property 1.13 a) and e); we get

$$
\begin{aligned}
1+d+\sum_{1}^{s-1} H(n+1) & >\varepsilon+1+d+\sum_{1}^{t-1} K(n+1) \\
& \geq \varepsilon+1+K(1)-1+\sum_{1}^{t-1}{ }_{n} K(n+1)
\end{aligned}
$$

hence

$$
e(H)-1>e(H)-e(K)+e(K)-1 \text { a contradiction. }
$$

Case 2. $i \leq s$

Let $d:=\delta_{i}(H(i))$. We observe that $\delta_{i}(H(i))=H(1)-2$ since $H$ is special. Being $H \cdot>K$ we have $H(i) \geq K(i)$ hence $d \geq \delta_{n}(H(n))$ for $n=$ $i, \cdots, s$ and $d \geq \delta_{n}(K(n))$ for $n=i, \cdots, t$ by Property 1.1 and Lemma 3.7.

We treat separately two subcases;

Subcase 2.1. $j>1$

We put

$$
A:=\sum_{1}^{i-2} n\left(\sum_{d+1}^{\infty} r\left(H(n+1)_{-1}\right)_{\langle n\rangle(r)}\right) \quad \text { and } B=\sum_{1}^{i-2} n\left(\sum_{d+1}^{\infty} r\left(K(n+1)_{-1}\right)_{\langle n\rangle(r)}\right) .
$$

We know that $H \cdot>K$, hence $H(n+1) \geq K(n+1)$ for $n=0, \cdots, i-2$, hence $A \geq B$ by Property 1.5 and Property 1.3. Adding this inequality to (4) and subtracting $d$ from both sides, we get

$$
\begin{aligned}
1 & +\sum_{1}^{i-2} n\left(\sum_{0}^{\infty}\left(H(n+1)_{-1}\right)_{\langle n\rangle(r)}\right)+\sum_{i=1}^{s-1} n\left(\sum_{0}^{d}\left(H(n+1)_{-1}\right)_{\langle n\rangle(r)}\right) \\
& >\varepsilon+1+\sum_{1}^{i-2}\left(\sum_{0}^{\infty}\left(K(n+1)_{-1}\right)_{\langle n\rangle(r)}\right)+\sum_{i=1}^{t-1} n\left(\sum_{0}^{d}\left(K(n+1)_{-1}\right)_{\langle n\rangle(r)}\right) .
\end{aligned}
$$

Now $H$ is special, hence $\delta_{n+1}(H(n+1))=d=H(1)-2$ for $n+1=i, \cdots$, $s-1$. We get

$$
\sum_{0}^{d} r\left(H(n+1)_{-1}\right)_{\langle n\rangle(r)}=H(n+1) \text { for } n+1=i, \cdots, s-1
$$

by Property $1.13 \mathrm{~d}$ )

and also 


$$
\sum_{0}^{d}\left(H(s)_{-1}\right)_{\langle s-1\rangle(r)}=H(s) \text {, being } j>1 \text {, by Property } 1.13 \text { a) } .
$$

Moreover Property $1.13 \mathrm{~d})$ also implies that $\sum_{0}^{\infty}\left(H(n+1)_{-1}\right)_{\langle n\rangle(r)}=H(n+1)$ for $n+1=1, \cdots, i-1$ being $H(n+1)$ a binomial coefficient.

Therefore the left hand side of (7a) is $e(H)-H(1)$, while the right hand side is greater than or equal to $\varepsilon+e(K)-K(1)$, again by Property 1.13 e). We deduce $e(H)-H(1)>e(H)-e(K)+e(K)-K(1)$. Being $H(1) \geq K(1)$, we get a contradiction.

Subcase 2.2. $j=1$

Let $p$ be the maximum integer such that $n(p)=n(2)+p-2$. Then of course $p \geq 2$ and

$$
H(s)_{-1}=\left(\begin{array}{c}
n(s)-1 \\
s-1
\end{array}\right)+\cdots+\left(\begin{array}{c}
n(p+1)-1 \\
p
\end{array}\right)+\left(\begin{array}{c}
n(2)+p-2 \\
p-1
\end{array}\right)
$$

by Property 1.11 .

By the inductive assumption, we may assume that the theorem holds for zero-sequences $H^{\prime}, K^{\prime}$ with socdeg $\left(H^{\prime}\right)<s$. By (1) and the equality

$$
\begin{aligned}
\left(H(s)_{-1}\right)_{\langle s-1\rangle(r)}= & \left(\begin{array}{c}
n(s)-1-r \\
s-1
\end{array}\right)+\cdots+\left(\begin{array}{c}
n(p+1)-1-r \\
p
\end{array}\right) \\
& +\left(\begin{array}{c}
n(2)+p-2-r \\
p-1
\end{array}\right)
\end{aligned}
$$

we may apply b) until $n(2)+p-2-r-(p-1)=n(2)-r-1>0$.

We get strict inequalities in formulae (2) i.e.

$$
e\left(\left(H_{-1}\right)_{\langle>(r)}\right)-1 \geq e\left(\left(K_{-1}\right)_{\langle>(r)}\right) \text { for every } r=1, \cdots, n(2)-1 .
$$

Subcase 2.2.1. $d \geq n(2)-1$

We sum (1), (2') for $r=1$ to $n(2)-1$, (2) for $r=n(2), \cdots, d$ and we get

$$
\sum_{0}^{d} e\left(\left(H_{-1}\right)_{\langle>(r)}\right)-n(2)+1>\varepsilon+\sum_{0}^{d} e\left(\left(K_{-1}\right)_{<>(r)}\right)
$$

hence as before

$$
\begin{aligned}
1+d+ & \sum_{1}^{s-1}\left(\sum_{0}^{d}\left(H(n+1)_{-1}\right)_{\langle n\rangle(r)}\right)-n(2)+1 \\
& >\varepsilon+1+d+\sum_{1}^{t-1}\left(\sum_{0}^{d}\left(K(n+1)_{-1}\right)_{\langle n\rangle(r)}\right)
\end{aligned}
$$


Now we proceed as in subcase 2.1 ; hence we put

$$
A:=\sum_{1}^{i-2} n\left(\sum_{d+1}^{\infty} r\left(H(n+1)_{-1}\right)_{\langle n\rangle(r)}\right) \text { and } B=\sum_{1}^{i-2} n\left(\sum_{d+1}^{\infty} r\left(K(n+1)_{-1}\right)_{\langle n\rangle(r)}\right) .
$$

We know that $H \cdot>K$, hence $H(n+1) \geq K(n+1)$ for $n+1=1, \cdots$, $i-1$, hence $A \geq B$ by Property 1.5 and Property 1.3.

Adding this inequality to $\left(4^{\prime}\right)$ and subtracting $d$ from both sides, we get

$$
\begin{array}{r}
1+\sum_{1}^{i-2} n\left(\sum_{0}^{\infty}\left(H(n+1)_{-1}\right)_{\langle n\rangle(r)}\right)+\sum_{i=1}^{s-1}\left(\sum_{0}^{d}\left(H(n+1)_{-1}\right)_{\langle n\rangle(r)}\right)-n(2)+1 \\
>\varepsilon+1+\sum_{1}^{i-2}\left(\sum_{0}^{\infty}\left(K(n+1)_{-1}\right)_{\langle n\rangle(r)}\right)+\sum_{i=1}^{t-1}\left(\sum_{0}^{d}\left(K(n+1)_{-1}\right)_{\langle n\rangle\langle r\rangle}\right) .
\end{array}
$$

Now $H$ is special, hence $\delta_{n+1}(H(n+1))=d=H(1)-2$ for $n+1=i, \cdots$, $s-1$. We use Property $1.13 \mathrm{~d}$ ) and we get

$$
\sum_{0}^{d}\left(H(n+1)_{-1}\right)_{\langle n\rangle(r)}=H(n+1) \text { for } n+1=i, \cdots, s-1 .
$$

Now $j=1$ and $d \geq n(2)-1$, hence $d>n(2)-2$; if $d=\delta_{s}(H(s))$ we cannot have $p=i$. Therefore

$$
\left.\sum_{0}^{d}\left(H(s)_{-1}\right)_{\langle s-1\rangle(r)}=H(s)+n(2)-n(1) \quad \text { by Property } 1.13 \mathrm{c}\right) .
$$

Moreover Property $1.13 \mathrm{~d}$ ) also implies that

$$
\left.\sum_{0}^{\infty}\left(H(n+1)_{-1}\right)_{\langle n\rangle(r)}\right)=H(n+1) \text { for } n+1=1, \cdots, i-1,
$$

being $H(n+1)$ a binomial coefficient. Therefore the left hand side of (7b) is

$$
e(H)+n(2)-n(1)-n(2)+1-H(1)=e(H)+1-n(1)-H(1),
$$

while the right hand side is greater than or equal to $\varepsilon+e(K)-K(1)$, again by Property 1.13 e). We deduce

$$
e(H)+1-n(1)-H(1)>e(H)-e(K)+e(K)-K(1) .
$$

Being $H(1) \geq K(1)$ and $n(1) \geq 1$, we get a contradiction

Subcase 2.2.2. $\quad d<n(2)-1$

We sum (1), (2') for $r=1$ to $d$ and we get

$$
\sum_{0}^{d} e\left(\left(H_{-1}\right)_{\langle>(r)}\right)-d>\varepsilon+\sum_{0}^{d} e\left(\left(K_{-1}\right)_{\langle>(r)}\right)
$$


hence as before

$$
\begin{aligned}
1+d+ & \sum_{1}^{s-1} n\left(\sum_{0}^{d}\left(H(n+1)_{-1}\right)_{\langle n\rangle(r)}\right)-d \\
& >\varepsilon+1+d+\sum_{1}^{t-1} n\left(\sum_{0}^{d} r\left(K(n+1)_{-1}\right)_{\langle n\rangle(r)}\right) .
\end{aligned}
$$

We have $n(2)-2 \leq \delta_{s}(H(s)) \leq d<n(2)-1$ hence $d=\delta_{s}(H(s))=n(2)-2$. This implies $p=i$, hence

$$
\sum_{0}^{d}\left(H(s)_{-1}\right)_{\langle s-1\rangle(r)}=H(s)+n(2)-n(1)-1 \quad \text { by Property } 1.13 .
$$

Now we proceed as in case 2.2.1 and we get

$$
\begin{aligned}
& e(H)-d+n(2)-n(1)-H(1)-1>e(H)-e(K)+e(K)-K(1) \text { hence } \\
& e(H)-n(2)+2+n(2)-n(1)-H(1)-1>e(H)-e(K)+e(K)-K(1) .
\end{aligned}
$$

As before, being $H(1) \geq K(1)$ and $n(1) \geq 1$, we get a contradiction

Theorem 3.10. Let $J$ be a zero-dimensional lex-segment ideal in $R=$ $k\left[x_{1}, \cdots, x_{h}\right], J \subseteq\left(x_{1}, \cdots, x_{h}\right)^{2}$; let $I$ be a homogeneous zero-dimensional ideal in $R^{\prime}=k\left[x_{1}, \cdots, x_{h^{\prime}}\right], I \subseteq\left(x_{1}, \cdots, x_{h^{\prime}}\right)^{2}$. Let $H_{R / J}$ be special, $H_{R / J} \cdot \geq$ $H_{R^{\prime} / I}, e(R / J) \geq e\left(R^{\prime} / I\right)$. Then $\nu(J) \geq \nu(I)$.

Proof. The assumptions imply that $H_{R / J}(1)=h$ and $H_{R^{\prime} / I}(1)=h^{\prime}$. If $\operatorname{socdeg}\left(H_{R / J}\right)>1$ then $H_{R / J} \cdot \geq H_{R^{\prime} / I}$ implies that $h \geq h^{\prime}$. If $\operatorname{socdeg}\left(H_{R / J}\right)$ $=1$ then $J=\left(x_{1}, \cdots, x_{h}\right)^{2}$, hence $e(R / J)=h+1 \geq e\left(R^{\prime} \mid I\right) \geq h^{\prime}+1$. Also in this case we get $h \geq h^{\prime}$. By Corollary 2.7 we may assume $I$ to be a lex-segment.

Now $\left(H_{R / J}\right)_{\langle\rangle(r)}$ is special for every $r$ by Proposition $\left.3.4 \mathrm{~b}\right)$. Moreover $\left(H_{R / J}\right)_{\langle\rangle(r)} \cdot \geq\left(H_{R / I}\right)_{\langle\rangle(r)}$ for every $r$, by Lemma 3.8. By repeated application of Main Lemma 3.9 we get $e\left(\left(H_{R / J}\right)_{\langle\rangle(r)}\right) \geq e\left(\left(H_{R^{\prime} / I}\right)_{\langle\rangle(r)}\right)$ for $r=1, \cdots, h^{\prime}$. Hence by Lemma 2.8,

$$
\begin{aligned}
& e\left(k\left[x_{1}, \cdots, x_{h}\right]_{\langle h-r+1\rangle} / J_{\langle h-r+1\rangle}\right) \geq e\left(k\left[x_{1}, \cdots, x_{h^{\prime}}\right]_{\left\langle h^{\prime}-r+1\right\rangle} \mid I_{\left\langle h^{\prime}-r+1\right\rangle}\right. \\
& \text { for } r=1, \cdots, h^{\prime} .
\end{aligned}
$$

Therefore

$$
\begin{aligned}
\nu(J) & =\sum_{1}^{h} e\left(k\left[x_{1}, \cdots, x_{h}\right]_{\langle h-r+1\rangle} / J_{\langle h-r+1\rangle}\right) \geq \sum_{1}^{h^{\prime}} e\left(k\left[x_{1}, \cdots, x_{h}\right]_{\langle h-r+1\rangle} / J_{\langle h-r+1\rangle}\right) \\
& \geq \sum_{1}^{h^{\prime}} e\left(k\left[x_{1}, \cdots, x_{h^{\prime}}\right]_{\left\langle h^{\prime}-r+1\right\rangle} \mid I_{\left\langle h^{\prime}-r+1\right\rangle}\right)=\nu(I) \quad \text { by Corollary } 2.10
\end{aligned}
$$


CoROLlary 3.11. With the same assumptions as in 3.10, if $h>h^{\prime}$ or indeg $(R / J)>$ indeg $(R / I)$, then $\nu(J)>\nu(I)$.

Proof. If $h>h^{\prime}$, then

$$
\begin{aligned}
\nu(J) & =\sum_{1}^{h} e\left(k\left[x_{1}, \cdots, x_{h}\right]_{\langle h-r+1\rangle} / J_{\langle h-r+1\rangle}\right)>\sum_{1}^{h^{\prime}} e\left(k\left[x_{1}, \cdots, x_{h}\right]_{\langle h-r+1\rangle} / J_{\langle h-r+1\rangle}\right) \\
& \geq \sum_{1}^{h^{\prime}} e\left(k\left[x_{1}, \cdots, x_{h^{\prime}}\right]_{\left\langle h^{\prime}-r+1\right\rangle} \mid I_{\left\langle h^{\prime}-r+1\right\rangle}=\nu(I) .\right.
\end{aligned}
$$

Now let $h=h^{\prime}$ and indeg $(R / J)>$ indeg $(R / I)$. We know that

$$
\begin{aligned}
& \operatorname{indeg}\left(k\left[x_{1}, \cdots, x_{h}\right]_{\langle 2\rangle} / J_{\langle 2\rangle}\right)=\operatorname{indeg}(R / J) \text { and } \\
& \text { indeg } \left.\left(k\left[x_{1}, \cdots, x_{h}\right]_{\langle 2\rangle} / I_{\langle 2\rangle}\right)=\operatorname{indeg}(R / I) \text { by Lemma } 3.3 \mathrm{a}\right) ;
\end{aligned}
$$

moreover it is clear that

$$
\begin{aligned}
& e\left(k\left[x_{1}, \cdots, x_{h}\right]_{\langle 2\rangle} / J_{\langle 2\rangle}\right)=\operatorname{indeg}\left(k\left[x_{1}, \cdots, x_{h}\right]_{\langle 2\rangle} / J_{\langle 2\rangle}\right) \quad \text { and } \\
& e\left(k\left[x_{1}, \cdots, x_{h}\right]_{\langle 2\rangle} / I_{\langle 2\rangle}\right)=\operatorname{indeg}\left(k\left[x_{1}, \cdots, x_{h}\right]_{\langle 2\rangle} / I_{\langle 2\rangle}\right), \quad \text { hence } \\
& e\left(k\left[x_{1}, \cdots, x_{h}\right]_{\langle 2\rangle} / J_{\langle 2\rangle}\right)>e\left(k\left[x_{1}, \cdots, x_{h}\right]_{\langle 2\rangle} / I_{\langle 2\rangle}\right) .
\end{aligned}
$$

We use again

$$
\begin{aligned}
\nu(J) & =\sum_{1}^{h} e\left(k\left[x_{1}, \cdots, x_{h}\right]_{\langle h-r+1\rangle} / J_{\langle h-r+1\rangle}\right) \geq \sum_{1}^{h} e\left(k\left[x_{1}, \cdots, x_{h}\right]_{\langle h-r+1\rangle} / J_{\langle h-r+1\rangle}\right) \\
& \geq \sum_{1}^{h} e\left(k\left[x_{1}, \cdots, x_{h}\right]_{\left\langle h^{\prime}-r+1\right\rangle} / I_{\left\langle h^{\prime}-r+1\right\rangle}\right)=\nu(I)
\end{aligned}
$$

and we see that one of the inequalities is strict

\section{§4. Main Theorems}

Definition. Given two positive integers $e, h$, with $e \geq h+1$, we define $t=t(e, h)$ as the unique integer such that

$$
\left(\begin{array}{c}
h+t-1 \\
t-1
\end{array}\right) \leq e<\left(\begin{array}{c}
h+t \\
t
\end{array}\right) \quad \text { and } \quad r=r(h, e):=e-\left(\begin{array}{c}
h+t-1 \\
t-1
\end{array}\right) .
$$

We remark that $t \geq 2$.

Lemma 4.1. Given two positive integers $e, h$, with $e \geq h+1$, there exists a unique zero-sequence $H=H(e, h)$, such that

a) $e(H)=e$

b) $H(1)=h$

c) $\operatorname{socdeg}(H)=t$ if $r>0, \operatorname{socdeg}(H)=t-1$ if $r=0$

d) $\operatorname{indeg}(H)=t$ 
Moreover such $H$ is special.

Proof. It is clear that

$$
H=\left(1, h,\left(\begin{array}{c}
h+1 \\
2
\end{array}\right), \cdots,\left(\begin{array}{c}
h+t-2 \\
t-1
\end{array}\right), r, 0\right)
$$

is the unique zero-sequence satisfying all the conditions

Definition. Given $e, h$ as before, we define $J=J(e, h)$ as the unique lex-segment ideal in $R=k\left[x_{1}, \cdots, x_{h}\right]$ such that $H_{R / J}=H(e, h)$.

Proposition 4.2. Given e, $h$ as before, we have

$$
\nu(J(e, h))=\left(\begin{array}{c}
h+t-1 \\
t
\end{array}\right)-r+r^{\langle t\rangle} .
$$

Proof. We know from Lemma 4.1 that

$$
H_{R / J}=\left(1, h,\left(\begin{array}{c}
h+1 \\
2
\end{array}\right), \cdots,\left(\begin{array}{c}
h+t-2 \\
t-1
\end{array}\right), r, 0\right) .
$$

We use Corollary 2.7 and the conclusion follows immediately

Definition. Given three positive integers $e, h, i$ with $e \geq h+1, i<$ $t(e, h)$, we define $s=s(e, h, i)$ as the unique integer such that

$$
\left(\begin{array}{c}
h+s-1 \\
s-1
\end{array}\right)-\left(\begin{array}{c}
h+s-i-1 \\
s-i-1
\end{array}\right) \leq e<\left(\begin{array}{c}
h+s \\
s
\end{array}\right)-\left(\begin{array}{c}
h+s-i \\
s-i
\end{array}\right) .
$$

We observe that such $s$ is well defined since the function

$$
f(n):=\left(\begin{array}{c}
h+n \\
n
\end{array}\right)-\left(\begin{array}{c}
h+n-i \\
n-i
\end{array}\right)
$$

is increasing. We define

$$
r=r(e, h, i):=e-\left(\begin{array}{c}
h+s-1 \\
s-1
\end{array}\right)+\left(\begin{array}{c}
h+s-i-1 \\
s-i-1
\end{array}\right) .
$$

Lemma 4.3. Given three positive integers $e, h, i$ with $e \geq h+1,2 \leq$ $i<t(e, h)$, there exists a unique zero-sequence $H=H(e, h, i)$ such that
a) $e(H)=e$
b) $H(1)=h$
c) $\operatorname{indeg}(H)=i$
d) $H(i)=\left(\begin{array}{c}h+i-1 \\ i\end{array}\right)-1$
e) $H$ is TVS. 
Moreover such $H$ is special.

Proof. We have

$$
\left(\begin{array}{c}
h+i-1 \\
i
\end{array}\right)-1=\left(\begin{array}{c}
h+i-2 \\
i
\end{array}\right)+\left(\begin{array}{c}
h+i-3 \\
i-1
\end{array}\right)+\cdots+\left(\begin{array}{c}
h-1 \\
1
\end{array}\right) .
$$

Let $H$ be the zero-sequence defined by

$$
\begin{aligned}
& H(n):=\left(\begin{array}{c}
h+n-1 \\
h
\end{array}\right) \quad \text { for } n=0, \cdots, i-1 ; \\
& H(i):=\left(\begin{array}{c}
h+i-1 \\
i
\end{array}\right)-1=\left(\begin{array}{c}
h+i-2 \\
i
\end{array}\right)+\left(\begin{array}{c}
h+i-3 \\
i-1
\end{array}\right)+\cdots+\left(\begin{array}{c}
h-1 \\
i
\end{array}\right) ; \\
& H(n+1)=H(n)^{\langle n\rangle} \quad \text { for } n=i, \cdots, s-2 \\
& H(s)=r ; \\
& H(n)=0 \text { for } n>s .
\end{aligned}
$$

We remark that

$$
H(n)=\left(\begin{array}{c}
h+n-1 \\
n
\end{array}\right)-\left(\begin{array}{c}
h+n-i-1 \\
n-i
\end{array}\right) \quad \text { for } n=i, \cdots, s-1,
$$

hence we have

$$
\sum_{0}^{s-1} H(n)=\left(\begin{array}{c}
h+s-1 \\
s-1
\end{array}\right)-\left(\begin{array}{c}
h+s-i-1 \\
s-i-1
\end{array}\right) .
$$

Therefore $e(H)=e$ and the other properties are clearly satisfied

Definition. Given $e, h, i$ as before, we define $J=J(e, h, i)$ as the unique lex-segment ideal in $R=k\left[x_{1}, \cdots, x_{h}\right]$ such that $H_{R / J}=H(e, h, i)$.

Proposition 4.4. Given $e, h, i$ as before, we have

$$
\nu(J(e, h, i))=1+\left(\begin{array}{c}
h+s-1 \\
s
\end{array}\right)-\left(\begin{array}{c}
h+s-i-1 \\
s-i
\end{array}\right)-r+r^{\langle s\rangle} .
$$

Proof. From Lemma 4.3 we know $H(e, h, i)$ hence $H_{R / J}$. The conclusion follows again from Corollary 2.7 and a simple computation.

Definition. Given four integers $e, h, i, p$ with $e \geq h+1, i<t(e, h)$, $0 \leq p<\left(\begin{array}{c}h+i-1 \\ i\end{array}\right)$, we define $\bar{p}$ as the least $i$-regular integer which is bigger than or equal to $\max \left(p,\left(\begin{array}{c}h+i-2 \\ i\end{array}\right)\right)$. Since $\left(\begin{array}{c}h+i-1 \\ i\end{array}\right)-1 \geq p$ and it is $i$-regular, we have $\bar{p} \leq\left(\begin{array}{c}h+i-1 \\ i\end{array}\right)-1$. 
Let $\left(\begin{array}{c}h+i-2 \\ i\end{array}\right)+\cdots+\left(\begin{array}{c}h+j-2 \\ j\end{array}\right)$ be the $i$-binomial expansion of $\bar{p}$ and let $s=s(e, h, i, p)$ be the unique integer such that

$$
\begin{gathered}
\left(\begin{array}{c}
h+s-1 \\
s-1
\end{array}\right)-\left(\begin{array}{c}
h+s-i+j-2 \\
s-i+j-2
\end{array}\right)+\left(\begin{array}{c}
h+j-2 \\
j-2
\end{array}\right) \\
\leq e<\left(\begin{array}{c}
h+s \\
s
\end{array}\right)-\left(\begin{array}{c}
h+s-i+j-1 \\
s-i+j-1
\end{array}\right)+\left(\begin{array}{c}
h+j-2 \\
j-2
\end{array}\right) .
\end{gathered}
$$

We observe that such $s$ is well defined since the function

$$
f(n):=\left(\begin{array}{c}
h+n \\
n
\end{array}\right)-\left(\begin{array}{c}
h+n-i+j-1 \\
n-i+j-1
\end{array}\right)+\left(\begin{array}{c}
h+j-2 \\
j-2
\end{array}\right)
$$

is increasing. We define

$$
r=r(e, h, i, p):=e-\left(\begin{array}{c}
h+s-1 \\
s-1
\end{array}\right)+\left(\begin{array}{c}
h+s-i+j-2 \\
s-i+j-2
\end{array}\right)-\left(\begin{array}{c}
h+j-2 \\
j-2
\end{array}\right) .
$$

LEMMA 4.5. Given four integers $e, h, i, p$ with $e \geq h+1,2 \leq i<t(e, h)$, $0 \leq p<\left(\begin{array}{c}h+i-1 \\ i\end{array}\right)$, there exists a unique zero-sequence $H=H(e, h, i, p)$ such that
a) $e(H)=e$
b) $H(1)=h$
c) $\operatorname{indeg}(H)=i$
d) $H(i)=\bar{p}$
e) $H$ is $T V S$

Moreover such $H$ is special.

Proof. We have $\bar{p}=\left(\begin{array}{c}h+\underset{i}{i}-2 \\ i\end{array}\right)+\cdots+\left(\begin{array}{c}h+j-2 \\ j\end{array}\right)$. Let $H$ be the zero-sequence defined by

$$
\begin{aligned}
& H(n):=\left(\begin{array}{c}
h+n-1 \\
n
\end{array}\right) \quad \text { for } n=0, \cdots, i-1 ; \\
& H(i):=\left(\begin{array}{c}
h+i-2 \\
i
\end{array}\right)+\cdots+\left(\begin{array}{c}
h+j-2 \\
j
\end{array}\right)=\left(\begin{array}{c}
h+i-1 \\
i
\end{array}\right)-\left(\begin{array}{c}
h+j-2 \\
j-1
\end{array}\right) \\
& H(n+1)=H(n)^{\langle n\rangle} \quad \text { for } n=i, \cdots, s-2, \\
& H(s)=r ; \\
& H(n)=0 \quad \text { for } n>s .
\end{aligned}
$$

We remark that

$$
H(n)=\left(\begin{array}{c}
h+n-1 \\
n
\end{array}\right)-\left(\begin{array}{c}
h+n-i+j-2 \\
n-i+j-1
\end{array}\right) \quad \text { for } n=i, \cdots, s-1,
$$


hence we have

$$
\begin{aligned}
& \sum_{0}^{s-1}{ }_{n} H(n)=\sum_{0}^{s-1} n\left(\begin{array}{c}
h+n-1 \\
n
\end{array}\right)-\sum_{i}^{s-1}\left(\begin{array}{c}
h+n-i+j-2 \\
n-i+j-1
\end{array}\right) \\
& =\sum_{0}^{s-1}\left(\begin{array}{c}
h+n-1 \\
n
\end{array}\right)-\sum_{0}^{s-1} n\left(\begin{array}{c}
h+n-i+j-2 \\
n-i+j-1
\end{array}\right)+\sum_{0}^{i-1} n\left(\begin{array}{c}
h+n-i+j-2 \\
n-i+j-1
\end{array}\right) \\
& =\left(\begin{array}{c}
h+s-1 \\
s-1
\end{array}\right)-\left(\begin{array}{c}
h+s-i+j-2 \\
s-i+j-2
\end{array}\right)+\left(\begin{array}{c}
h+j-2 \\
j-2
\end{array}\right) .
\end{aligned}
$$

Therefore $e(H)=e$ and the other properties are clearly satisfied

Definition. Given $e, h, i, p$ as before, we define $J=J(e, h, i, p)$ as the unique lex-segment ideal in $R=k\left[x_{1}, \cdots, x_{h}\right]$ such that $H_{R / J}=H(e, h$, $i, p)$.

Proposition 4.6. Given $e, h, i, p$ as before, we have

$$
\begin{aligned}
& \nu(J(e, h, i, p)) \\
& \quad=\left(\begin{array}{c}
h+j-2 \\
j-1
\end{array}\right)+\left(\begin{array}{c}
h+s-1 \\
s
\end{array}\right)-\left(\begin{array}{c}
h+s-i+j-2 \\
s-i+j-1
\end{array}\right)-r+r^{\langle s\rangle} .
\end{aligned}
$$

Proof. From Lemma 4.5 we know $H(e, h, i, p)$ hence $H_{R / J}$. The conclusion follows again from Corollary 2.7 and a simple computation

In the following when we say that a homogeneous polynomial ideal $I$ has multiplicity $e$, we mean that $I$ is an ideal of a polynomial ring $R$ such that $R / I$ has multiplicity $e$.

Definition. Given a positive integer $e$ we define $\mathscr{F}(e)$ to be the family of zero-dimensional homogeneous polynomial ideals with multiplicity $e$. Given two positive integers $e, h$ with $e \geq h+1$ we define $\mathscr{F}(e, h)$ to be the family of zero-dimensional homogeneous ideals $I$ in $R:=k\left[x_{1}, \cdots, x_{h}\right]$ with $I \subseteq\left(x_{1}, \cdots, x_{h}\right)^{2}$ and multiplicity $e$. Given three positive integers $e$, $h$, $i$, with $e \geq h+1,2 \leq i \leq t(e, h)$ (see definition before Lemma 4.1), we define $\mathscr{F}(e, h, i)$ to be the family of zero-dimensional homogeneous ideals $I$ in $R:=k\left[x_{1}, \cdots, x_{h}\right]$ with $I \subseteq\left(x_{1}, \cdots, x_{h}\right)^{2}$, multiplicity $e$ and indeg $(R / I)$ $=i$. Given four positive integers $e, h, i, p$, with $e \geq h+1,2 \leq i \leq t(e, h)$ and $0 \leq p \leq\left(\begin{array}{c}h+i+1 \\ i\end{array}\right)$, we define $\mathscr{F}(e, h, i, p)$ to be the family of zerodimensional homogeneous ideals $I$ in $R:=k\left[x_{1}, \cdots, x_{h}\right]$ with $I \subseteq\left(x_{1}, \cdots\right.$, $\left.x_{h}\right)^{2}$, multiplicity $e$, indeg $(R / I)=i$ and $H_{R / I}(i)=p$.

TheOREM 4.7. 1) The ideal $\left(x_{1}, \cdots, x_{e-1}\right)^{2}$ of $k\left[x_{1}, \cdots, x_{e-1}\right]$ is in $\mathscr{F}(e)$ and $\nu(I) \leq \nu\left(\left(x_{1}, \cdots, x_{e-1}\right)^{2}\right)=\left(\begin{array}{l}e \\ 2\end{array}\right)$ for every ideal $I$ in $\mathscr{F}(e)$. 
2) The ideal $J(e, h)$ is in $\mathscr{F}(e, h)$ and $\nu(I) \leq \nu(J(e, h))=\left(\begin{array}{c}h+t-1 \\ t\end{array}\right)-r+r^{\langle t\rangle}$ for every ideal I in $\mathscr{F}(e, h)$.

3) The ideal $J(e, h, i)$ is in $\mathscr{F}(e, h, i)$ and

$$
\begin{aligned}
\nu(I) \leq \nu(J(e, h, i))=1+\left(\begin{array}{c}
h+s-1 \\
s
\end{array}\right)- & \left(\begin{array}{c}
h+s-i-1 \\
s-i
\end{array}\right)-r+r^{\langle s\rangle} \\
& \text { for every ideal I in } \mathscr{F}(e, h, i) .
\end{aligned}
$$

4) The ideal $J(e, h, i, p)$ is in $\mathscr{F}(e, h, i, p)$ and

$$
\begin{aligned}
\nu(I) \leq \nu(J(e, h, i, p))= & \left(\begin{array}{c}
h+j-2 \\
j-1
\end{array}\right)+\left(\begin{array}{c}
h+s-1 \\
s
\end{array}\right)-\left(\begin{array}{c}
h+s-i+j-2 \\
s-i+j-1
\end{array}\right) \\
& -r+r^{\langle s\rangle} \quad \text { for every ideal I in } \mathscr{F}(e, h, i, p) .
\end{aligned}
$$

Proof. The ideals $\left(x_{1}, \cdots, x_{e-1}\right)^{2}, J(e, h), J(e, h, i)$ and $J(e, h, i, p)$ have special Hilbert functions by Lemma 4.1, Lemma 4.3, Lemma 4.5. Moreover $H_{R / J} \cdot \geq H_{R^{\prime} / I}$ by the very definition of $J$ (here $J$ means $\left(x_{1}, \cdots, x_{e-1}\right)^{2}$ or $J(e, h)$ or $J(e, h, i)$ or $J(e, h, i, p))$. Hence the conclusion follows from Theorem 3.10

Theorem 4.8. 1) $\nu(J(e, h, i, p)) \leq \nu(J(e, h, i, q))$ if $p \leq q$

2) $\nu(J(e, h, i, p)) \leq \nu(J(e, h, i)) \leq \nu(J(e, h))$

3) $\nu(J(e, h, i))<\nu(J(e, h, i+1))$

4) $\nu(J(e, h))<\nu(J(e, h+1))$

5) $\quad \nu(J(e, h, i, p)) \leq \nu(J(e+1, h, i, p)) ; \quad \nu(J(e, h, i)) \leq \nu(J(e+1, h, i))$; $\nu(J(e, h)) \leq \nu(J(e+1, h))$

Proof. Along the lines of the proof of Theorem 4.7, the conclusions follow from Theorem 3.10 and Corollary 3.11

ExAMPLES 1) Let $J=\left(x^{2}, x y, x z^{3}, y^{4}, y^{3} z^{2}, y^{2} z^{3}, y z^{4}, z^{5}\right)$; then $H:=H_{R / J}$ $=(1,3,4,5,4,0)$. Let $I=\left(x^{2}, x y, x z^{2}, y^{5}, y^{4} z, y^{3} z^{2}, y^{2} z^{3}, y z^{4}, z^{5}\right)$; then $K:=$ $H_{R / J}=(1,3,4,4,5,0)$. Then $e(R / J)=e(R / I)=17$ and $H \cdot \geq K ; H_{\langle\rangle}=(1$, $2,1,1,0), K_{\langle\rangle}=(1,2,1,1,1,0)$, hence $e\left(H_{\langle\rangle}\right)<e\left(K_{\langle\rangle}\right)$. This shows that in the Main Lemma 3.9 we cannot delete the assumption of speciality on $H$.

Moreover $\nu(J)=8, \nu(I)=9$; this shows that also in Theorem 3.10 we cannot delete the assumption of speciality on $H$.

2) Let $R:=k\left[x_{1}, x_{2}, x_{3}\right], J=J(64,3,2)$; by Proposition $4.4, \nu(J)=18$. Let $J^{\prime}=J(64,3,3,4)$; by Proposition $4.6, \nu\left(J^{\prime}\right)=17$. This shows that $\nu(J(e, h, i)) \leq \nu(J(e, h, i+1, p))$ does not hold for every $p$. 


\section{§5. Applications}

\section{Perfect ideals in regular local rings}

In the following we are going to use some well-known properties of local algebra, in particular the theory of reductions. All the necessary details can be found for instance in Herrmann-Ikeda-Orbanz (1988). Let $(R, \mathfrak{m}, k)$ be a regular local ring and $\mathfrak{a}$ an ideal in $R$. Let $i=\operatorname{indeg}(\mathfrak{a})$ be the maximum integer $n$ such that $\mathfrak{a} \subseteq \mathfrak{m}^{n}$.

Definition. Given a positive integer $e$ we define

$\operatorname{CM}(e)$ to be the family of perfect ideals $a$ in regular local rings $(R, \mathfrak{m}, k)$ with $e(R / \mathfrak{a})=e$. Given two positive integers $e, h$ with $e \geq h+1$, we define

$\mathrm{CM}(e, h)$ to be the family of perfect codimension $h$ ideals $\mathfrak{a}$ in regular local rings $(R, \mathfrak{m}, k)$ with $\mathfrak{a} \subseteq \mathfrak{m}^{2}$ and $e(R / \mathfrak{a})=e$. Given three positive integers $e, h, i$, with $e \geq h+1,2 \leq i \leq t(e, h)$ (see definition before Lemma 4.1), we define

$\mathrm{CM}(e, h, i)$ to be the family of perfect codimension $h$ ideals $a$ in regular local rings $(R, \mathfrak{m}, k)$ with $\mathfrak{a} \subseteq \mathfrak{m}^{2}$, indeg $(\mathfrak{a})=i$ and $e(R / \mathfrak{a})=e$. Given four positive integers $e, h, i, p$, with $e \geq h+1,2 \leq i \leq t(e, h)$ and $0 \leq p \leq\left(\begin{array}{c}h+i-1 \\ i\end{array}\right)$, we define

$\operatorname{CM}(e, h, i, p)$ to be the family of perfect codimension $h$ ideals $a$ in regular local rings $(R, \mathfrak{m}, k)$ with $\mathfrak{a} \subseteq \mathfrak{m}^{2}$, indeg $(\mathfrak{a})=i, H_{R / \mathfrak{a}}(i)=p$ and $e(R / \mathfrak{a})=e$.

In the following theorem we use the notations of Section 4 .

TheOREM 5.1. 1) Let $(R, \mathrm{nt}, k)$ be a regular local ring of dimension $e-1$. The ideal $\mathrm{m}^{2}$ is in $\operatorname{CM}(e)$ and $\nu(\mathfrak{a}) \leq \nu\left(\mathfrak{m}^{2}\right)=\left(\begin{array}{l}e \\ 2\end{array}\right)$ for every ideal $\mathfrak{a}$ in $\operatorname{CM}(e)$.

2) For every ideal a in $\operatorname{CM}(e, h), \nu(\mathfrak{a}) \leq\left(\begin{array}{c}h+t-1 \\ t\end{array}\right)-r+r^{\langle t\rangle}$.

3) For every ideal a in $\operatorname{CM}(e, h, i)$,

$$
\nu(\mathfrak{a}) \leq 1+\left(\begin{array}{c}
h+s-1 \\
s
\end{array}\right)-\left(\begin{array}{c}
h+s-i-1 \\
s-i
\end{array}\right)-r+r^{\langle s\rangle} .
$$

4) For every ideal a in $\operatorname{CM}(e, h, i, p)$,

$$
\nu(\mathfrak{a}) \leq\left(\begin{array}{c}
h+j-2 \\
j-1
\end{array}\right)+\left(\begin{array}{c}
h+s-1 \\
s
\end{array}\right)-\left(\begin{array}{c}
h+s-i+j-2 \\
s-i+j-1
\end{array}\right)-r+r^{\langle s\rangle} .
$$


Proof. It is well-known that there exists a minimal reduction $\underline{x}:=$ $x_{1}, \cdots, x_{N-h}$ of $m \bmod a$, such that indeg $(\mathfrak{a})=\operatorname{indeg}(\mathfrak{a}+(\underline{x}) /(\underline{x})$ ) (EliasIarrobino (1987)).

Let $\bar{R}:=R /(\underline{x}) ; \overline{\mathfrak{a}}:=\mathfrak{a}+(\underline{x}) /(\underline{x}) ; \overline{\mathfrak{m}}:=\mathfrak{m} /(\underline{x})$. Then $(\bar{R}, \overline{\mathfrak{m}}, k)$ is a regular local ring of dimension $h$ and $G:=\operatorname{gr}_{\text {m } / / \bar{a}}(\bar{R} / \overline{\mathfrak{a}})=k\left[x_{1}, \cdots, x_{h}\right] / I$, where $I=i n_{\mathfrak{m}}(\overline{\mathfrak{a}})$ is a homogeneous ideal contained in $\left(x_{1}, \cdots, x_{h}\right)^{2}$. We get $\nu(\mathfrak{a})$ $=\nu(\overline{\mathfrak{a}})$ since $x$ is a regular sequence $\bmod \mathfrak{a}$ and $\nu(\overline{\mathfrak{a}}) \leq \nu(I)$, since every standard basis is a basis. Clearly indeg $(\mathfrak{a})=\operatorname{indeg}(\overline{\mathfrak{a}})=\operatorname{indeg}(I), h=$ $\operatorname{codim}(I)$ and $e(R / \mathfrak{a})=e(\bar{R} / \overline{\mathfrak{a}})=e\left(k\left[x_{1}, \cdots, x_{h}\right] / I\right)$.

Hence the conclusions follows from Theorem 4.7

Remark 1. It is clear that the conclusions of Theorem 5.1 apply as well to perfect homogeneous ideals of polynomial rings.

Remark 2. Let $R=k[x, y]$ and $I=(x) \cap(x, y)^{n}$. Then $e(R / I)=1$ while $\nu(I)=n$. This shows that Theorem 5.1 does not extend to non perfect ideals.

Codimension 2 perfect ideals.

Proposition 5.2. Let $(R, \mathfrak{m}, k)$ be a regular local ring and $\mathfrak{a}$ a perfect codimension 2 ideal in $R$. Then $\nu(\mathfrak{a}) \leq$ indeg $(\mathfrak{a})+1$.

Proof. As in the proof of Theorem 5.1 we have $\nu(\mathfrak{a}) \leq \nu(I)$, where $I$ is a homogeneous zero-dimensional ideal of $R=k\left[x_{1}, x_{2}\right]$. By Corollary 2.7 we get $\nu(I) \leq \nu(J)$ where $J$ is a lex-segment ideal of $R=k\left[x_{1}, x_{2}\right]$. We apply Corollary 2.10 and we get $\nu(J)=e\left(R_{\langle 1\rangle} / J_{\langle 1\rangle}\right)+e\left(R_{\langle 2\rangle} / J_{\langle 2\rangle}\right)$. But $e\left(R_{\langle 1\rangle} / J_{\langle 1\rangle}\right)=1$ and clearly $e\left(R_{\langle 2\rangle} / J_{\langle 2\rangle}\right)=\operatorname{indeg}(J)$.

Since indeg $(J)=$ indeg $(\mathfrak{a})$, the conclusion follows

Powers of maximal ideals.

Proposition 5.3. a) Let $I$ be a perfect codimension $h$ ideal of a regular local ring $(R, \mathfrak{m})$. Assume that $\nu(I)=\left(\begin{array}{c}h+t-1 \\ t\end{array}\right)$ and $e(R / I)=$ $\left(\begin{array}{c}h+t-1 \\ t-1\end{array}\right)$. Then indeg $(I) \geq t$.

b) If moreover $\operatorname{dim}(R / I)=0$, then $I=\mathfrak{m}^{t}$.

Proof. a) If indeg $(I)=i<t$, then $I \in \mathrm{CM}(e, h, i)$, hence $\nu(I) \leq(J(e$, $h, i))$ by Theorem 5.1 and $\nu(J(e, h, i))<\nu(J(e, h, t)) \leq \nu(J(e, h))$ by Theorem 4.82 ) and 3). Now $\nu(J(e, h))=\left(\begin{array}{c}h+t-1 \\ t\end{array}\right)$ by Proposition 4.2. 
b) By a) we get $I \subseteq \mathfrak{m}^{t}$. It $I \subset \mathfrak{m}^{t}$ then $e(R / I)=l(R / I)>l\left(R / \mathfrak{m}^{t}\right)=$ $\left(\begin{array}{c}h+t-1 \\ t-1\end{array}\right)$, where $l$ denotes length, a contradiction.

Asymptotic formulae.

In the following if $\left\{a_{n}\right\}$ and $\left\{b_{n}\right\}$ are sequences of positive real numbers, we write $\left\{a_{n}\right\} \sim\left\{b_{n}\right\}$ if $\lim _{n \rightarrow \infty} a_{n} / b_{n}=1$. We are interested in the asymptotic behaviour of the sequences $\nu(J(e, h))$ and $\nu(J(e, h, i))$, where we let $e$ tend to infinity, while $h$ and $i$ are kept fixed.

TheOREM 5.4. a) $\{\nu(J(e, h))\} \sim\left\{(h / \sqrt[h]{h !}) e^{1-1 / h}\right\}$.

b) Let $k:=h-1$; then $\{J(e, h, i)\} \sim\left\{\sqrt[k]{i}(k / \sqrt[k]{k !}) e^{1-1 / k}\right\}$.

Proof. a) We first compute the asymptotic behaviour of the subsequences $\left\{\nu_{e_{t}}\right\}$ of $\left\{\nu_{e}\right\}:=\{\nu(J(e, h))\}$, where $e_{t}:=\left(\begin{array}{c}h+t-1 \\ t-1\end{array}\right)$. We know from Proposition 4.2 that $\nu_{e_{t}}=\left(\begin{array}{c}h+t-1 \\ t\end{array}\right)=\left(\begin{array}{c}t+h-1 \\ h-1\end{array}\right)$, hence $t \nu_{e_{t}}=h e_{t}$. Since $\nu_{e_{t}}$ is a polynomial in $t$ with leading term $t^{h-1} /(h-1)$ !, it is clear that $\left\{\nu_{e_{t}}\right\} \sim\left\{t^{h-1} /(h-1) !\right\}$, hence $\left\{\nu_{e_{t}}\right\} \sim\left\{\left(h e_{t}\right)^{h-1} /(h-1) !\left(\nu_{e_{t}}\right)^{h-1}\right\}$ which implies $\left\{\left(\nu_{e_{t}}\right)^{h}\right\} \sim\left\{\left(h e_{t}\right)^{h-1} /(h-1) !\right\}=\left\{h^{h} e_{t}^{h-1} / h !\right\}$, whence $\left\{\nu_{e_{t}}\right\} \sim\left\{(h / \sqrt[h]{h !}) e_{t}^{1-1 / h}\right\}$.

In general, given $e$ we let $t:=t(e, h)$ (see Section 4). Proposition 4.2 implies $\nu_{e_{t}} \leq \nu_{e} \leq \nu_{e_{t+1}}$. Let $\varphi_{e}:=(h / \sqrt[h]{h !}) e^{1-1 / h}$; then $\varphi_{e_{t}} \leq \varphi_{e} \leq \varphi_{e_{t+1}}$ hence $\left(\nu_{e_{t}} / \varphi_{e_{t}}\right)\left(\varphi_{e_{t}} / \varphi_{e_{t+1}}\right)=\nu_{e_{t}} / \varphi_{e_{t+1}} \leq \nu_{e} / \varphi_{e} \leq \nu_{e_{t+1}} / \varphi_{e_{t}}=\left(\nu_{e_{t+1}} / \varphi_{e_{t+1}}\right)\left(\varphi_{e_{t+1}} / \varphi_{e_{t}}\right)$. Now it suffices to prove that $\left\{\varphi_{e_{t}}\right\} \sim\left\{\varphi_{e_{t+1}}\right\}$. For, it is enough to show that $\left\{e_{t}\right\}$ $\sim\left\{e_{t+1}\right\}$ and this is true since they are polynomials in $t$ with the same leading term.

b) We first compute the asymptotic behaviour of the subsequence $\left\{\nu_{e_{s}}\right\}$ of $\left\{\nu_{e}\right\}:=\{\nu(J(e, h, i))\}$, where $e_{s}:=\left(\begin{array}{c}h+s-1 \\ s-1\end{array}\right)-\left(\begin{array}{c}h+s-i-1 \\ s-i-1\end{array}\right)$.

We know from Proposition 4.4 that

$$
\nu_{e_{s}}=1+\left(\begin{array}{c}
h+s-1 \\
s
\end{array}\right)-\left(\begin{array}{c}
h+s-i-1 \\
s-1
\end{array}\right) .
$$

It is easy to see that $e_{s}$ and $\nu_{e_{s}}$ are polynomials in $s$ with leading term $\left(i s^{h-1}\right) /(h-1)$ ! and $\left(i s^{h-2}\right) /(h-2)$ ! respectively. We get $\left\{(h-1) e_{s}\right\} \sim\left\{s \nu_{e_{s}}\right\}$, therefore $\left\{\nu_{e_{s}}\right\} \sim\left\{i\left[(h-1) e_{s}\right]^{h-2} /(h-2) !\left(\nu_{e_{s}}\right)^{h-2}\right\}$, which implies

$$
\left\{\left(\nu_{e_{s}}\right)^{h-1}\right\} \sim\left\{i\left[(h-1) e_{s}\right]^{h-2} /(h-2) !\right\}=\left\{i(h-1)^{h-1} e_{s}^{h-2} /(h-1) !\right\},
$$

whence $\left\{\nu_{e_{s}}\right\}=\left\{\sqrt[k]{i}(k / \sqrt[k]{k !}) e_{s}^{1-1 / k}\right\}$

The general case can be handled as in a) 
Ideals of points in $\mathbb{P}^{n}$.

Let $I$ be the defining ideal of a set $X:=\left\{P_{1}, \cdots, P_{e}\right\}$ of $e$ distinct points in $\mathbb{P}^{n}$. In the following proposition we use the notations of section 4

Proposition 5.5. a) $\nu(I) \leq\left(\begin{array}{c}h+t-1 \\ t\end{array}\right)-r+r^{\langle t\rangle}$.

b) If $i$ is the minimal degree of hypersurfaces containing $X$, then

$$
\nu(I) \leq 1+\left(\begin{array}{c}
h+s-1 \\
s
\end{array}\right)-\left(\begin{array}{c}
h+s-i-1 \\
s-i
\end{array}\right)-r+r^{\langle s\rangle} .
$$

c) If $i$ is the minimal degree of hypersurfaces containing $X$ and $p$ is the number of independent conditions imposed by $X$ on the linear system of hypersurfaces of degree $i$, then

$$
\nu(I) \leq\left(\begin{array}{c}
h+j-2 \\
j-1
\end{array}\right)+\left(\begin{array}{c}
h+s-1 \\
s
\end{array}\right)-\left(\begin{array}{c}
h+s-i+j-2 \\
s-i+j-1
\end{array}\right)-r+r^{\langle s\rangle} .
$$

d) All the preceding bounds are sharp.

Proof. a), b), c) are immediate consequences of Theorem 5.1 since $I$ is a perfect codimension $h$ ideal in $R:=k\left[x_{0}, \cdots, x_{h}\right]$ such that $e(R / I)=e$.

d) The bounds are attained by the monomial ideals $J(e, h), J(e, h, i)$, $J(e, h, i, p)$ respectively. By a result of Hartshorne (see Geramita-GregoryRoberts (1986)) monomial ideals in $k\left[x_{1}, \cdots, x_{h}\right]$ can be lifted to ideals of distinct points in $\mathbb{P}^{n}$, with the same number of generators, same indeg and multiplicity

ExAmples. Let $h=3, e=10$. Then $J(10,3)=\left(x_{1}, x_{2}, x_{3}\right)^{3}$ (see Section 4) and $\nu(J(10,3))=10$. We construct a set $X:=\left\{P_{1}, \cdots, P_{10}\right\}$ of 10 distinct points in $\mathbb{P}^{3}$, whose defining ideal has 10 generators.

$$
J(10,3)=\left(x_{1}, x_{2}, x_{3}\right)^{3}=\left(x_{1}^{3}, x_{1}^{2} x_{2}, x_{1}^{2} x_{3}, x_{1} x_{2}^{2}, x_{1} x_{2} x_{3}, x_{1} x_{3}^{2}, x_{2}^{3}, x_{2}^{2} x_{3}, x_{2} x_{3}^{2}, x_{3}^{3}\right) .
$$

This ideal can be lifted for instance to the following radical ideal $I$ in $k\left[x_{0}, x_{1}, x_{2}, x_{3}\right]$ :

$$
\begin{aligned}
I= & \left(x_{1}\left(x_{1}-x_{0}\right)\left(x_{1}-2 x_{0}\right), x_{1}\left(x_{1}-x_{0}\right) x_{2}, x_{1}\left(x_{1}-x_{0}\right) x_{3}, x_{1} x_{2}\left(x_{2}-x_{0}\right),\right. \\
& x_{1} x_{2} x_{3}, x_{1} x_{3}\left(x_{3}-x_{0}\right), x_{2}\left(x_{2}-x_{0}\right)\left(x_{2}-2 x_{0}\right), x_{2}\left(x_{2}-x_{0}\right) x_{3}, x_{2} x_{3}\left(x_{3}-x_{0}\right), \\
& \left.x_{3}\left(x_{3}-x_{0}\right)\left(x_{3}-2 x_{0}\right)\right) .
\end{aligned}
$$

This is the defining ideal of the following 10 points

$$
\begin{aligned}
& P_{1}=(1,0,0,0) ; P_{2}=(1,0,0,1) ; P_{3}=(1,0,0,2) ; P_{4}=(1,0,1,0) ; \\
& P_{5}=(1,0,1,1) ; P_{6}=(1,0,2,0) ; P_{7}=(1,1,0,0) ; P_{8}=(1,1,0,1) ;
\end{aligned}
$$




$$
P_{9}=(1,1,1,0) ; P_{10}=(1,2,0,0) .
$$

Let $h=3, e=10, i=2$. Then $J(10,3,2)=\left(x_{1}^{2}, x_{1} x_{2}^{2}, x_{1} x_{2} x_{3}, x_{1} x_{3}^{2}, x_{2}^{3}, x_{2}^{2} x_{3}\right.$, $\left.x_{2} x_{3}^{2}, x_{3}^{4}\right)$ see section 4$)$ and $\nu(J(10,3,2))=8$. As before we construct a set $X:=\left\{P_{1}, \cdots, P_{10}\right\}$ of 10 distinct points on a quadric of $\mathbb{P}^{3}$, whose defining ideal has 8 generators. The points are

$$
\begin{aligned}
& P_{1}=(1,0,0,0) ; P_{2}=(1,0,0,1) ; P_{3}=(1,0,0,2) ; P_{4}=(1,0,0,3) ; \\
& P_{5}=(1,0,1,0) ; P_{6}=(1,0,1,1) ; P_{7}=(1,0,2,0) ; P_{8}=(1,1,0,0) ; \\
& P_{9}=(1,1,0,1) ; P_{10}=(1,1,1,0) .
\end{aligned}
$$

They lie on the quadric $x_{1}\left(x_{1}-x_{0}\right)=0$.

\section{Gröbner Bases of homogeneous Ideals}

For an introduction to the subject see for instance Robbiano (1988). If $I$ is an ideal in $k\left[x_{1}, \cdots, x_{n}\right]$ and $\sigma$ is a term-ordering, then $I$ has a unique reduced Gröbner Basis $G_{\sigma}(I)$ with respect to $\sigma$. The cardinality of $G_{\sigma}(I)$ is the same as that of the monomial ideal $L t_{\sigma}(I)$. Therefore, if $I$ is a zero-dimensional ideal of $k\left[x_{1}, \cdots, x_{n}\right]$, the cardinality of $G_{\sigma}(I)$ is subjected to the bounds obtained in Corollary 2.7 and Theorem 4.7.

For example if $(f, g, h)$ is a regular sequence of homogeneous elements in $k[x, y, z]$ such that $\operatorname{deg}(f)=2, \operatorname{deg}(g)=3$, $\operatorname{deg}(h)=5$, and $I$ is the ideal generated by $\{f, g, h\}$, then $e(R / I)=30$, the Hilbert function is $(1,3$, $5,6,6,5,3,1,0)$ and the corresponding lex-segment ideal is

$$
J=\left(x^{2}, x y^{2}, x y z^{2}, x z^{4}, y^{5}, y^{4} z^{2}, y^{3} z^{3}, y^{2} z^{5}, y z^{6}, z^{8}\right) .
$$

Since $\nu(J)=10$, we deduce from Corollary 2.7 that every reduced Gröbner basis of $I$ has cardinality smaller than or equal to 10 .

\section{$A$ remark on tangent cones}

Let $I$ be a perfect ideal of a regular local ring $(R, \mathfrak{n})$. Very often, if the ring $(A=R / I, \mathfrak{m}=\mathfrak{n} / I)$ has numerical characters, which are extremal with respect to some general inequality, the tangent cone $\operatorname{gr}_{\mathrm{m}}(A)$ is again Cohen-Macaulay (see Sally (1977), Elias (1986), Elias-Iarrobino (1987), Rossi-Valla (1988)). We show that this is not the case in our situation.

Let $A:=k[[x, y, z, t]] / I$ where $I$ is the defining ideal of the union of the monomial curves with parametric equations $\left\{t^{5}, t^{6}, t^{14}, 0\right\}$ and $\left\{0, s, 0, s^{3}\right\}$. Then $I=\left(y^{4}-x^{2} z-y w, x^{4}-y z, x^{2} y^{3}-z^{2}, z w, x w, y^{3} w-w^{2}\right)$ hence $\nu(I)=$ $6=\nu(J(6,3,2))$. The tangent cone is given by the equations $\left(y w, y z, z^{2}\right.$, 
$\left.z w, x w, w^{2}, x^{3} z, x y^{5}\right)$ and the Hilbert function is $H_{A}=(1,4,4,5,5,6,6, \cdots)$. Therefore $\operatorname{gr}_{\mathrm{m}}(A)$ is not Cohen-Macaulay since $H_{A}$ is not strictly increasing up to the multiplicity.

Multiplicity, Codimension, Maximum number of generators

$\begin{array}{ccccc}(4,3 ; 6) & (5,3 ; 6) & (6,3 ; 6) & (7,3 ; 7) & (8,3 ; 7) \\ (9,3 ; 8) & (10,3 ; 10) & (11,3 ; 10) & (12,3 ; 10) & (13,3 ; 10) \\ (14,3 ; 11) & (15,3 ; 11) & (16,3 ; 11) & (17,3 ; 12) & (18,3 ; 12) \\ (19,3 ; 13) & (20,3 ; 15) & (21,3 ; 15) & (22,3 ; 15) & (23,3 ; 15) \\ (24,3 ; 15) & (25,3 ; 16) & (26,3 ; 16) & (27,3 ; 16) & (28,3 ; 16) \\ (29,3 ; 17) & (30,3 ; 17) & (31,3 ; 17) & (32,3 ; 18) & (33,3 ; 18) \\ (34,3 ; 19) & (35,3 ; 21) & (36,3 ; 21) & (37,3 ; 21) & (38,3 ; 21) \\ (39,3 ; 21) & (40,3 ; 21) & (41,3 ; 22) & (42,3 ; 22) & (43,3 ; 22) \\ (44,3 ; 22) & (45,3 ; 22) & (46,3 ; 23) & (47,3 ; 23) & (48,3 ; 23) \\ (49,3 ; 23) & (50,3 ; 24) & (51,3 ; 24) & (52,3 ; 24) & (53,3 ; 25) \\ (54,3 ; 25) & (55,3 ; 26) & (56,3 ; 28) & (57,3 ; 28) & (58,3 ; 28) \\ (59,3 ; 28) & (60,3 ; 28) & (61,3 ; 28) & (62,3 ; 28) & (63,3 ; 29) \\ (64,3 ; 29) & (65,3 ; 29) & (66,3 ; 29) & (67,3 ; 29) & (68,3 ; 29) \\ (69,3 ; 30) & (70,3 ; 30) & (71,3 ; 30) & (72,3 ; 30) & (73,3 ; 30) \\ (74,3 ; 31) & (75,3 ; 31) & (76,3 ; 31) & (77,3 ; 31) & (78,3 ; 32) \\ (79,3 ; 32) & (80,3 ; 32) & (81,3 ; 33) & (82,3 ; 33) & (83,3 ; 34) \\ (84,3 ; 36) & (85,3 ; 36) & (86,3 ; 36) & (87,3 ; 36) & (88,3 ; 36) \\ (89,3 ; 36) & (90,3 ; 36) & (91,3 ; 36) & (92,3 ; 37) & (93,3 ; 37) \\ (94,3 ; 37) & (95,3 ; 37) & (96,3 ; 37) & (97,3 ; 37) & (98,3 ; 37) \\ (99,3 ; 38) & (100,3 ; 38) & (101,3 ; 38) & (102,3 ; 38) & (103,3 ; 38) \\ (104,3 ; 38) & (105,3 ; 39) & (106,3 ; 39) & (107,3 ; 39) & (108,3 ; 39) \\ (109,3 ; 39) & (110,3 ; 40) & (111,3 ; 40) & (112,3 ; 40) & (113,3 ; 40) \\ (114,3 ; 41) & (115,3 ; 41) & (116,3 ; 41) & (117,3 ; 42) & (118,3 ; 42) \\ (119,3 ; 45) & (120,3 ; 45) & (121,3 ; 45) & (122,3 ; 45) & (123,3 ; 45) \\ (124,3 ; 45) & (125,3 ; 45) & (126,3 ; 45) & (127,3 ; 45) & (128,3 ; 45) \\ (129,3 ; 46) & (130,3 ; 46) & (131,3 ; 46) & (132,3 ; 46) & (133,3 ; 46) \\ (134,3 ; 46) & (135,3 ; 46) & (136,3 ; 46) & (137,3 ; 47) & (138,3 ; 47) \\ (139,3 ; 47) & (140,3 ; 47) & (141,3 ; 47) & (142,3 ; 47) & (143,3 ; 47) \\ (144,3 ; 48) & (145,3 ; 48) & (146,3 ; 48) & (147,3,48) & (148,3 ; 48) \\ (149,3 ; 48) & (150,3 ; 49) & (151,3 ; 49) & (152,3 ; 49) & (153,3 ; 49) \\ (154,3 ; 49) & (155,3 ; 50) & (156,3 ; 50) & (157,3 ; 50) & (158,3 ; 50) \\ (159,3 ; 51) & (160,3 ; 51) & (161,3 ; 51) & (162,3 ; 52) & (163,3 ; 52)\end{array}$


Multiplicity, Codimension, Initial degree, Maximum number of generators

$\begin{array}{ccccc}(4,3,2 ; 6) & (5,3,2 ; 6) & (6,3,2 ; 6) & (7,3,2 ; 7) & (8,3,2 ; 7) \\ (9,3,2 ; 8) & (10,3,2 ; 8) & (11,3,2 ; 8) & (12,32 ; 8) & (13,3,2 ; 9) \\ (14,3,2 ; 9) & (15,3,2 ; 9) & (16,3,2 ; 10) & (17,3,2 ; 10) & (18,3,2 ; 10) \\ (19,3,2 ; 10) & (20,3,2 ; 10) & (21,3,2 ; 11) & (22,3,2 ; 11) & (23,3,2 ; 11) \\ (23,3,2 ; 11) & (25,3,2 ; 12) & (26,3,2 ; 12) & (27,3,2 ; 12) & (28,3,2 ; 12) \\ (29,3,2 ; 12) & (30,3,2 ; 12) & (31,3,2 ; 13) & (32,3,2 ; 13) & (33,3,2 ; 13) \\ (34,3,2 ; 13) & (35,3,2 ; 13) & (36,3,2 ; 14) & (37,3,2 ; 14) & (38,3,2 ; 14) \\ (39,3,2 ; 14) & (40,3,2 ; 14) & (41,3,2 ; 14) & (42,3,2 ; 14) & (43,3,2 ; 15) \\ (44,3,2 ; 15) & (45,3,2 ; 15) & (46,3,2 ; 15) & (47,3,2 ; 15) & (48,3,2 ; 15) \\ (49,3,2 ; 16) & (50,3,2 ; 16) & (51,3,2 ; 16) & (52,3,2 ; 16) & (53,3,2 ; 16) \\ (54,3,2,16) & (55,3,2 ; 16) & (56,3,2 ; 16) & (57,3,2 ; 17) & (58,3,2 ; 17) \\ (59,3,2 ; 17) & (60,3,2 ; 17) & (61,3,2 ; 17) & (62,3,2 ; 17) & (63,3,2 ; 17) \\ (64,3,2 ; 18) & (65,3,2 ; 18) & (66,3,2 ; 18) & (67,3,2 ; 18) & (68,3,2 ; 18) \\ (69,3,2 ; 18) & (70,3,2 ; 18) & (71,3,2 ; 18) & (72,3,2 ; 18) & (73,3,2 ; 19) \\ (74,3,2 ; 19) & (75,3,2 ; 19) & (76,3,2 ; 19) & (77,3,2 ; 19) & (78,3,2 ; 19) \\ (79,3,2 ; 19) & (80,3,2 ; 19) & (81,3,2 ; 20) & (82,3,2 ; 20) & (83,3,2 ; 20) \\ (84,3,2 ; 20) & (85,3,2 ; 20) & (86,3,2 ; 20) & (87,3,2 ; 20) & (88,3,2 ; 20) \\ (89,3,2 ; 20) & (90,3,2 ; 20) & (91,3,2 ; 21) & (92,3,2 ; 21) & (93,3,2 ; 21) \\ (94,3,2 ; 21) & (95,3,2 ; 21) & (96,3,2 ; 21) & (97,3,2 ; 21) & (98,3,2 ; 21) \\ (99,3,2 ; 21) & (100,3,2 ; 22) & (101,3,2 ; 22) & (102,3,2 ; 22) & (103,3,2 ; 22) \\ (104,3,2 ; 22) & (105,3,2 ; 22) & (106,3,2 ; 22) & (107,3,2 ; 22) & (108,3,2 ; 22) \\ (109,3,2 ; 22) & (110,3,2 ; 22) & (111,3,2 ; 23) & (112,3,2 ; 23) & (113,3,2 ; 23) \\ (114,3,2 ; 23) & (115,3,2 ; 23) & (116,3,2 ; 23) & (117,3,2 ; 23) & (118,3,2 ; 23) \\ (119,3,2 ; 23) & (120,3,2 ; 23) & (121,3,2 ; 24) & (122,3,2 ; 24) & (123,3,2 ; 24) \\ (124,3,2 ; 24) & (125,3,2 ; 24) & (126,3,2 ; 24) & (127,3,2 ; 24) & (128,3,2 ; 24) \\ (129,3,2 ; 24) & (130,3,2 ; 24) & (131,3,2 ; 24) & (132,3,2 ; 24) & (133,3,2 ; 25) \\ (134,3,2 ; 25) & (135,3,2 ; 25) & (136,3,2 ; 25) & (137,3,2 ; 25) & (139,3,2 ; 25) \\ (139,3,2 ; 25) & (140,3,2 ; 25) & (141,3,2 ; 25) & (142,3,2 ; 25) & (143,3,2 ; 25) \\ (144,3,2 ; 26) & (145,3,2 ; 26) & (146,3,2 ; 26) & (147,3,2 ; 26) & (148,3,2 ; 26) \\ (149,3,2 ; 26) & (150,3,2 ; 26) & (151,3,2 ; 26) & (152,3,2 ; 26) & (153,3,2 ; 26) \\ (154,3,2 ; 26) & (155,3,2 ; 26) & (156,3,2 ; 26) & (157,3,2 ; 27) & (158,3,2 ; 27) \\ (159,3,2 ; 27) & (160,3,2 ; 27) & (161,3,2 ; 27) & (162,3,2 ; 27) & (163,3,2 ; 27)\end{array}$




\section{REFERENCES}

[1] Berman, D., The number of generators of a colength $N$ ideal in a power series ring, J. Algebra, 73 (1981), 156-166.

[2] Boratynski, M., Eisenbud, D., Rees, D., On the number of generators of ideals in local Cohen-Macaulay rings, J. Algebra, 57 (1979), 77-81.

[ 3 ] Briançon, J., Iarrobino, A., Dimension of the punctual Hilbert scheme, J. Algebra, 55 (1978), 536-544.

[4] Elias, J., A sharp bound for the minimal number of generators of perfect height two ideals, Manuscripta Math., 55 (1986), 93-99.

[5] Elias, J., Iarrobino, A., Extremal Gorenstein algebras of codimension three; the Hilbert function of a Cohen-Macaulay local algebra, J. Algebra, 110 (1987), 344356.

[6] Geramita, A., Gregory, D., Roberts, L., Monomial ideals and points in projective space, J. Pure Appl. Algebra, 40 (1986), 33-62.

[7] Green, M., Restrictions of linear series to hyperplanes, and some results of Macaulay and Gotzmann, Algebraic Curves and Projective Geometry Proceedings, Tronto (1988), Springer L.N. in Mathematics 1389.

[ 8 ] Herrmann, M., Ikeda, S., Orbanz, U., Equimultiplicity and blowing up, Berlin, Heiderberg, New York: Springer (1988).

[ 9 ] Macaulay, F. S., Some properties of enumeration in the theory of modular systems, Proc. London Math. Soc., 26 (1927), 531-555.

[10] Robbiano, L., Introduction to the theory of Gröbner bases, Queen's Papers in Pure and Appl. Math., $\mathrm{N}^{\circ} \mathbf{8 0}$, Vol. V (1988).

[11] Robbiano, L., Valla, G., Basic Commutative Algebra, In preparation.

[12] Rossi, M. E., Valla, G., Multiplicity and t-isomultiple ideals, Nagoya Math. J., 110 (1988), 81-111.

[13] Sally, J. D., Bounds for number of generators of Cohen-Macaulay ideals, Pac. J. Math., 63 (1976), 517-520.

[14] —-, On the associated graded ring of a local Cohen-Macaulay ring, J. Math. Kyoto Univ., 17 (1977), 19-21.

[15] - Numbers of generators of ideals in local rings, Lecture Notes in Pure and Applied Mathematics, 35, New York: Marcel Dekker (1978).

[16] Stanley, R. P., Hilbert functions of graded algebras, Adv. in Math., 28 (1978), $57-83$.

[17] Valla, G., Generators of ideals and multiplicities, Comm. Algebra, 15 (1981), 1541-1549.

J. Elias

Department de Algebra i Geometria

Universitat de Barcelona

08007 Barcelona

Spain

L. Robbiano

G. Valla

Dipartimento di Matematica

Universita' di Genova

Via L. B. Alberti 416132 Genova

Italy 